Review

\title{
Extremely Re-Rich Molybdenite from Porphyry Cu-Mo-Au Prospects in Northeastern Greece: Mode of Occurrence, Causes of Enrichment, and Implications for Gold Exploration
}

\author{
Panagiotis Voudouris ${ }^{1, *}$, Vasilios Melfos ${ }^{2}$, Paul G. Spry ${ }^{3}$, Luca Bindi ${ }^{4}$, Robert Moritz ${ }^{5}$, \\ Melissa Ortelli ${ }^{5}$ and Tamara Kartal $^{6}$
}

1 Department of Mineralogy-Petrology, University of Athens, Athens 15784, Greece

2 Department of Mineralogy, Petrology and Economic Geology, Aristotle University of Thessaloniki, Thessaloniki 54124, Greece; E-Mail: melfosv@geo.auth.gr

3 Department of Geological and Atmospheric Sciences, 253 Science I, Iowa State University, Ames, IA 50011-3212, USA; E-Mail: pgspry@iastate.edu

4 Department of Earth Sciences, University of Florence, Florence I-50121, Italy;

E-Mail: luca.bindi@unifi.it

5 Department of Mineralogy, University of Geneva, Geneva CH-1205, Switzerland;

E-Mails: robert.moritz@unige.ch (R.M.); melissa.ortelli@unige.ch (M.O.)

6 Institute of Mineralogy-Petrology, University of Hamburg, Hamburg D-20146, Germany;

E-Mail: kartaltamara@aol.de

* Author to whom correspondence should be addressed; E-Mail: voudouris@geol.uoa.gr; Tel.: +30-201-727-4129; Fax: +30-210-727-4883.

Received: 19 February 2013; in revised form: 1 April 2013 / Accepted: 19 April 2013 /

Published: 2 May 2013

\begin{abstract}
Extremely Re-rich molybdenite occurs with pyrite in sodic-calcic, sodic-sericitic and sericitic-altered porphyritic stocks of granodioritic-tonalitic and granitic composition in the Sapes-Kirki-Esymi, Melitena and Maronia areas, northeastern Greece. Molybdenite in the Pagoni Rachi and Sapes deposits is spatially associated with rheniite, as well as with intermediate $(\mathrm{Mo}, \mathrm{Re}) \mathrm{S}_{2}$ and $(\mathrm{Re}, \mathrm{Mo}) \mathrm{S}_{2}$ phases, with up to $46 \mathrm{wt} \% \mathrm{Re}$. Nanodomains and/or microinclusions of rheniite may produce the observed Re enrichment in the intermediate molybdenite-rheniite phases. The extreme Re content in molybdenite and the unique presence of rheniite in porphyry-type mineralization, combined with preliminary geochemical data $(\mathrm{Cu} / \mathrm{Mo}$ ratio, Au grades) may indicate that these deposits have affinities with $\mathrm{Cu}-\mathrm{Au}$ deposits, and should be considered potential targets for gold mineralization in
\end{abstract}


the porphyry environment. In the post-subduction tectonic regime of northern Greece, the extreme Re and Te enrichments in the magmatic-hydrothermal systems over a large areal extent are attributed to an anomalous source (e.g., chemical inhomogenities in the mantle-wedge triggered magmatism), although local scale processes cannot be underestimated.

Keywords: molybdenite; rhenium; rheniite; porphyry; post-subduction; Greece

\section{Introduction}

Rhenium has an average concentration of $<1 \mathrm{ppb}$ in the Earth's crust, and there are few places where it is enriched to ore grades. It is among the most expensive metals and is used principally as a high-temperature super-alloy in jet engines. It is mainly obtained as a by-product of molybdenum and copper refinement from $\mathrm{Cu}$-Mo porphyry and sediment-hosted deposits. Re-bearing molybdenite with $>1 \mathrm{wt} \% \mathrm{Re}$ is rare, but has been found in porphyry-style $\mathrm{Cu}-\mathrm{Mo}$ deposits [1-3]. Several $\mathrm{Re}-\mathrm{Mo}-\mathrm{Cu}$ sulfides, mostly of scientific interest, are described from magmatic $\mathrm{Cu}-\mathrm{Ni}$ deposits in mafic-ultramafic complexes in Sweden [4], Canada [5], Montana [6], Karelia [7], and Finland [8]. Recently, native rhenium was identified in Precambrian chromite ores hosted in serpentinized dunites-harzburgites of the Ukrainian shield [9]. Dzhezkazganite $\left(\mathrm{ReMoCu}_{2} \mathrm{PbS}_{6}\right)$ is the main Re-bearing phase in chalcocite ores from the sediment-hosted Dzhezkazgan deposit, Kazakhstan [10]. An Os-Cu rheniite [( $\left.\mathrm{Re}, \mathrm{Cu}, \mathrm{Os}, \mathrm{Fe}) \mathrm{S}_{2}\right]$ occurs in bornite-rich ore in sandstone at Voronov Bor, Karelia, Russia [11], whereas rheniite $\left(\operatorname{ReS}_{2}\right)$ was initially discovered in fumarolic sublimates from Kudryavy volcano, Kurile Islands, Russia, which is its type locality [12].

Rheniite was also discovered in the Pagoni Rachi and Konos porphyry prospects, northeastern Greece $[13,14]$, which are the first known occurrences of this mineral in porphyry-style mineralization. The Pagoni Rachi and Konos prospects are part of a cluster of porphyry-type occurrences in northeastern Greece (the others include Maronia, Myli and Melitena), characterized by molybdenite with the highest Re contents yet reported from a porphyry-type deposit (up to 4.7 wt \% Re) [2,3,13-19]. The study of [13] indicated that the Re-rich molybdenite from Pagoni Rachi crystallized as the $2 \mathrm{H}$ polytype and not the $3 \mathrm{R}$ polytype, as previously hypothesized [1,20], which suggests that Re concentration does not correlate with a specific polytype. In addition, the almost perfect linear correlation between the Mo and Re content of molybdenite from porphyry-type prospects in northeastern Greece supports the previously proposed concept [13,21-23] that Re substitutes for Mo in the structure of molybdenite.

The Re content of molybdenite is controlled by several factors, including the composition of the ore-forming solution and the host rock, the nature and source of the host rock, the total amount of molybdenite in a given deposit, and the physicochemical conditions of ore formation [19,23]. Rhenium grades for porphyry $\mathrm{Cu}-\mathrm{Au}$ and porphyry Mo deposits are comparable (in the range of 0.01 to $0.6 \mathrm{~g} / \mathrm{t}$ ), suggesting that there is no difference between the Re content of Mo-dominant and $\mathrm{Cu}$-dominant porphyry deposits of comparable tonnage [3]. The recently discovered Merlin Mo-Re zone, Australia, an unusual vein-replacement deposit, probably genetically related to the Mt. Dore IOCG deposit, is the world's highest grade molybdenum-rhenium deposit, the current grades averaging $0.6 \mathrm{wt} \%$ Mo and 
$10 \mathrm{~g} / \mathrm{t} \mathrm{Re}$, which is much higher than the majority of porphyry Mo deposits where molybdenum grades are of the order of 0.1 to $0.2 \mathrm{wt} \%$ Mo [24,25].

A Re-enrichment in molybdenite also occurs in several other porphyry-style deposits/prospects in northern Greece, including Skouries of Chalkidiki (up to $1000 \mathrm{~g} / \mathrm{t}$, average $=900 \mathrm{~g} / \mathrm{t}$, [19]), Sardes, Limnos Island (up to $5200 \mathrm{~g} / \mathrm{t}$, average $=3785 \mathrm{~g} / \mathrm{t}$ ) and Fakos, Limnos Island (up to $2220 \mathrm{~g} / \mathrm{t}$, average $=1396 \mathrm{~g} / \mathrm{t}$ ) and Stypsi, Lesvos Island (up to $10,600 \mathrm{~g} / \mathrm{t}$, average $=2460 \mathrm{~g} / \mathrm{t}$ ), but these $\operatorname{Re}$ values are much lower than those from molybdenite in porphyry prospects in northeastern Greece [14]. By contrast, molybdenite from reduced intrusion-related Mo-W systems (Kimmeria deposit, Xanthi; Axioupolis prospect, Kilkis; Plaka deposit Lavrion; Serifos island) and shear zone-related $\mathrm{Cu}-\mathrm{Au}-\mathrm{Bi}-\mathrm{Mo}$ mineralization (Stanos deposit, Chalkidiki) is almost Re-free ([14], Figure 1).

This study evaluates earlier data on Re-enriched molybdenites from porphyry-type deposits in northeastern Greece, presents new information concerning recent molybdenite discoveries, and highlights the reasons for Re-enrichment. On the deposit scale, associated ore mineralogy and geochemistry is tested as a tool for Re and Au-enrichment and the use of these elements in the exploration of Re-bearing porphyry deposits in Greece.

Figure 1. Location of molybdenite-bearing mineralization in Greece (modified after [14]).

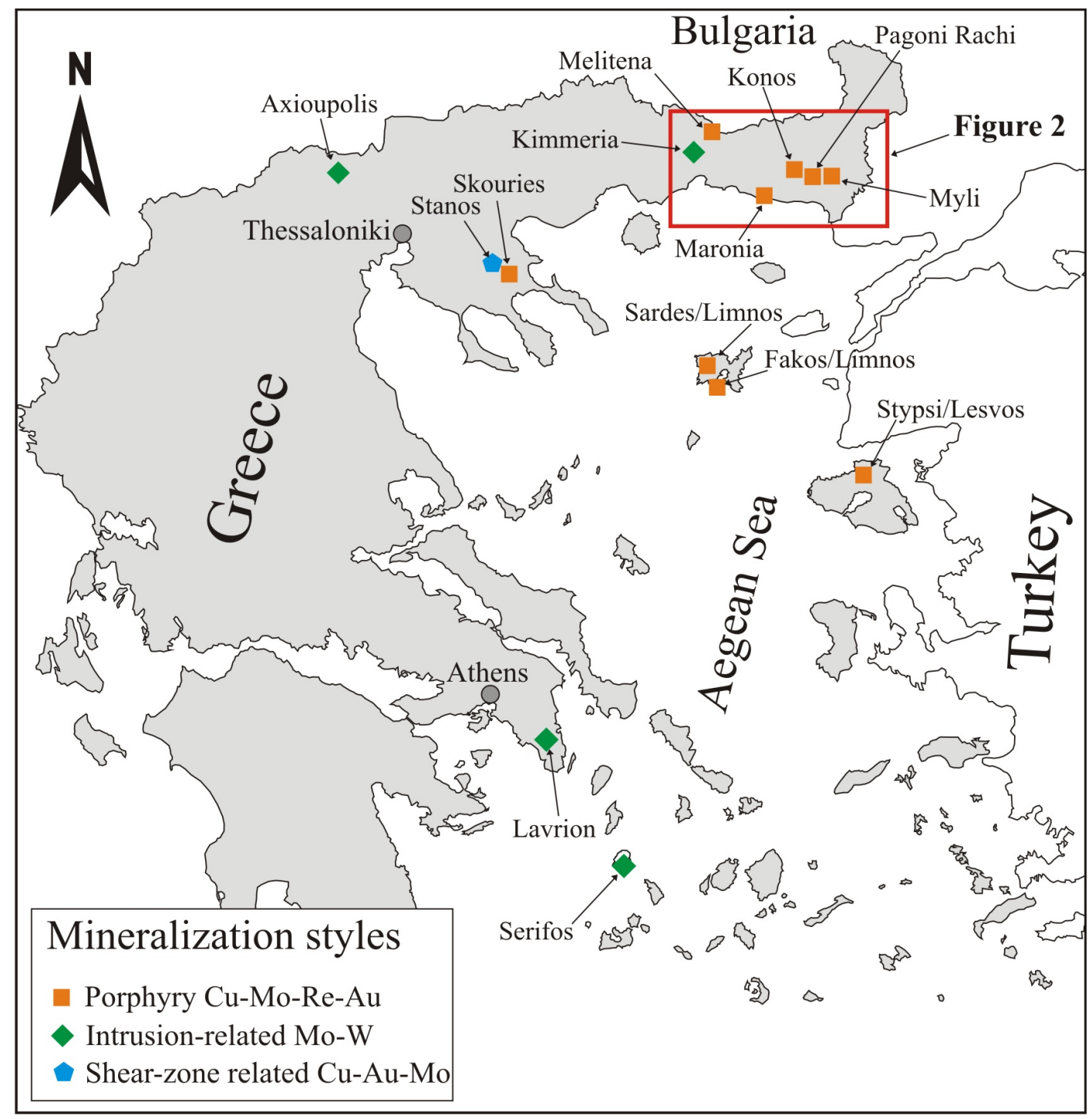




\section{Methods}

Seventy-eight thin and polished thin sections of host rocks and sulfide assemblages were studied with an optical microscope and a JEOL JSM 5600 (Tokyo, Japan) scanning electron microscope equipped with back-scattered imaging capabilities, at the Department of Mineralogy and Petrology, University of Athens, Greece. The composition of molybdenite was determined with a Cameca SX 100 (Gennevilliers, France) wavelength-dispersive electron microprobe at the Department of Mineralogy and Petrology, University of Hamburg, Germany. Operating conditions were: $20 \mathrm{kV}$ and $20 \mathrm{nA}$, counting time 20 to $60 \mathrm{~s}$, beam diameter $<2 \mu \mathrm{m}$. The following X-ray lines were used: $\operatorname{Mo} L \beta, \operatorname{Re} M \alpha$, $\mathrm{CuK \alpha}, \mathrm{Fe} K \alpha$ and $\mathrm{S} K \alpha$. Rhenium metal (for $\mathrm{Re}$ ), pyrite and chalcopyrite (for $\mathrm{Fe}$ and $\mathrm{S}$ ), and molybdenite (for Mo), were used as standards. The chemical composition of 87 molybdenite and rheniite grains was also determined using a JEOL JXA 8200 electron microprobe at Iowa State University, Ames, IA, USA. The concentrations of the major and minor elements were determined at an accelerating voltage of $15 \mathrm{kV}$ and a beam current of $15 \mathrm{nA}$, with $10 \mathrm{~s}$ as the counting time $(5 \mathrm{~s}$ on each background), beam diameter $<2 \mu \mathrm{m}$. The standards employed were metallic rhenium and molybdenum for $\mathrm{Re}$ and $\mathrm{Mo}$, chalcopyrite for $\mathrm{Cu}$, and synthetic pyrite for $\mathrm{S}$. Representative samples from unaltered rocks hosting porphyry-type mineralization were analyzed for major and trace elements by X-ray fluorescence techniques with a Phillips PW 1220 (Amsterdam, The Netherlands) instrument in the Institute of Mineralogy and Petrology, University of Hamburg. Bulk ore analyses of 44 mineralized samples were performed at ACME Labs, Vancouver, Canada using aqua regia digestion and Ultratrace ICP-MS analysis.

\section{Regional Geological and Metallogenetic Setting}

The Hellenides, part of the Alpine-Himalayan orogeny, were formed from the Late Jurassic to the present above the Hellenic subduction by the off-scraping of crustal units from the Pelagonian continent in the north and then the Pindos Ocean; the Apulian platform and eastern Mediterranean Ocean further south were subducted below Eurasia after the closure of the Vardar Ocean in the Late Cretaceous period [26-28]. Since Early Cretaceous, a single subduction zone without slab detachment was active and consumed both oceanic and continental lithospheric mantles beneath the Aegean Sea $[26,27,29,30]$.

In the Rhodope Massif (Bulgaria and Greece), Late Cretaceous-Tertiary exhumation of deep metamorphic rocks along detachment faults resulted in the formation of metamorphic core complexes, supra-detachment sedimentary basins, and widespread Late Eocene to Early Miocene basic to felsic magmatism [31] (Figure 2). Early cooling/exhumation (syn-orogenic exhumation) in the northern and eastern part of the Rhodope Massif was initiated in the Maastrichtian to Early Paleocene (up to $\sim 40 \mathrm{Ma})$ [27,32]. Jolivet et al. [27] and Wüthrich [32] distinguished two following stages of widespread extension and metamorphic core complex formation in the Rhodope Massif: a period of peak-cooling/exhumation from $\sim 40$ Ma to Late Eocene-Early Oligocene (35-33 Ma) without migration of volcanic centers (suggesting that the subduction zone was stationary), followed by a second period (Oligocene-Miocene) of fast back-arc extension that is considered to be the result of slab retreat (post-orogenic slab-rollback driven extension). The two extensional phases may indicate two successive 
"push-pull" cycles [33], which were caused by the accretion/subduction of continental and oceanic lithosphere fragments within the Aegean subduction zone [32]. The first phase of core complex formation in the Rhodope Massif (peak-cooling/exhumation between 40 and $33 \mathrm{Ma}$ ) is related to the first "push-pull" cycle (subduction of the Pindos Ocean following accretion of the Pelagonian continental block to the Moesian platform and previous accreted units, e.g., Drama continent, Vardar Ocean), and to voluminous bimodal magmatism and volcanism in the Rhodope Massif [32,34]. The Late Eocene-Oligocene (e.g., 35-25 Ma) magmatism in the Rhodope Massif was caused by convective removal of the lithospheric mantle (lithospheric delamination) and subsequent upwelling of the asthenosphere [31]. The upwelling asthenosphere provided the heat for hydrous melting of a heterogeneously enriched subcontinental mantle, resulted in the weakening of the crust, and may have produced the accelerated generation of the metamorphic core complexes in the Rhodope Massif [32,34,35]. The plutonic rocks (mostly granite to monzonite), and calc-alkaline to shoshonitic and ultra-K volcanic associations have geochemical and isotope affinities with supra-subduction rocks [31,36,37]. The second phase of core complex related extension in the Rhodope Massif was coeval with the accretion and subduction of a continental block composed of several microcontinents (Gavrovo-Tripolitsa, Olympos, Ionian, Apulian), and the subsequent subduction of the Mediterranean Sea [32]. Slab rollback (since about 35-30 Ma ago) is consistent with the progressive southward migration of magmatic activity from the Tertiary in the Rhodope Massif to the present in the South Aegean volcanic arc, and for the development of the Aegean subduction zone [26,27,32,36,38].

Figure 2. Simplified geological map of the southeastern part of the Rhodope Massif and location of major porphyry $\mathrm{Cu}-\mathrm{Mo}-\mathrm{Au}$ and epithermal $\mathrm{Cu}-\mathrm{Au}-\mathrm{Ag}-\mathrm{Te}$ deposits (after [39]). Abbreviations: $\mathrm{Me}=$ Melitena; $\mathrm{Mr}=$ Maronia; $\mathrm{Ki}=$ Kimmeria; $\mathrm{PH}=$ Perama Hill; $\mathrm{Ko}=$ Konos; SD = St Demetrios; V = Viper; PR = Pagoni Rachi; My = Myli. Sapes-Kirki-Esymi area (blue square). Abbreviations: $\mathrm{RM}=$ Rhodope Massif, $\mathrm{CRB}=$ Circum-Rhodope Belt.

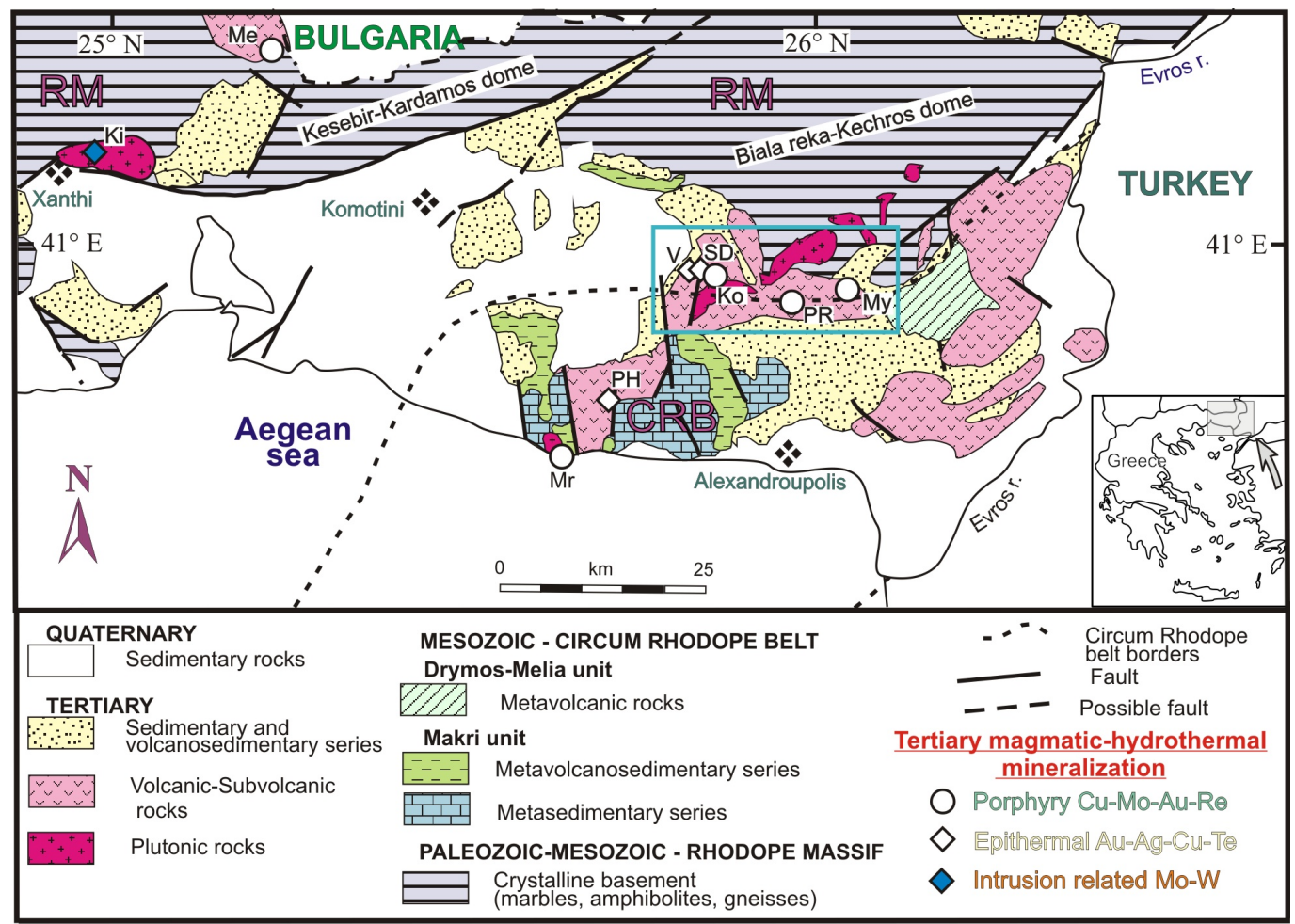


The Palaeogene magmatism of the central and eastern Rhodope Massif culminated at about 30-35 Ma and was accompanied by abundant ore-forming hydrothermal activity $[31,40]$. Several styles of mineralization in northern Greece (base- and precious-metal porphyry, reduced intrusion-related systems, epithermal, Carlin-type) are genetically related to arc-related magmatic rocks, and are, in part, controlled by exhumation structures of the Rhodope metamorphic core complex [31,39,41-45]. Porphyry $\mathrm{Cu}-\mathrm{Mo}-\mathrm{Au}$ systems that contain Re-rich molybdenite in northeastern Greece are genetically related to microgranite (Maronia) and granodiorite porphyries (Konos, Pagoni Rachi, Myli) [39,42,46-48] (Figure 2).

\section{Local Geology and Petrography}

\subsection{Sapes-Kirki-Esymi}

The Sapes-Kirki-Esymi area (about $300 \mathrm{~km}^{2}$; Figure 2), represents a very promising area for future $\mathrm{Au}$ and Re exploration/exploitation in northeastern Greece. It includes at least three major porphyry systems, Konos (Sapes), Pagoni Rachi (Kirki) and Myli (Esymi) [42]. The area consists of Tertiary volcanosedimentary rocks, as well as subvolcanic rocks of intermediate to felsic composition. The porphyry $\mathrm{Cu}-\mathrm{Mo}-\mathrm{Au}$ systems are multi-centered with earlier granodiorite-tonalite-hosted porphyry mineralization $[47,48]$, followed by microdiorite-hosted mineralization $\left({ }^{40} \mathrm{Ar} /{ }^{39} \mathrm{Ar}\right.$ age for hydrothermal biotite of $32.0 \pm 0.5 \mathrm{Ma}$; [48]). Late quartz-feldspar porphyry dikes exposed at Sapes are considered to be equivalent to the microgranite porphyry in the Maronia area. The host rocks for the Re-enriched molybdenite mineralization at Konos, Pagoni Rachi and Myli are the granodiorite-tonalite porphyries (Figure 3). They contain phenocrysts of plagioclase, amphibole, biotite, rare quartz and magnetite in a matrix of microlitic quartz, orthoclase and minor plagioclase. They display high-K calc-alkaline affinity and subalkaline character (Figure 3).

Figure 3. (a) Total alkali vs. silica diagram of igneous rocks hosting Re-rich porphyry $\mathrm{Cu}-\mathrm{Mo}-\mathrm{Au}$ mineralization in northeastern Greece (e.g., granodiorite-tonalite porphyry, microgranite porphyry and granodiorite porphyry). Whole-rock geochemical analyses are from [42,49] for Pagoni Rachi and Maronia and this study (Konos, Myli, Melitena). Rock fields are from [50], whereas the boundary between the alkaline and subalkaline fields is from [51]. (b) Q-ANOR plot used to classify the intrusive rocks after [52].
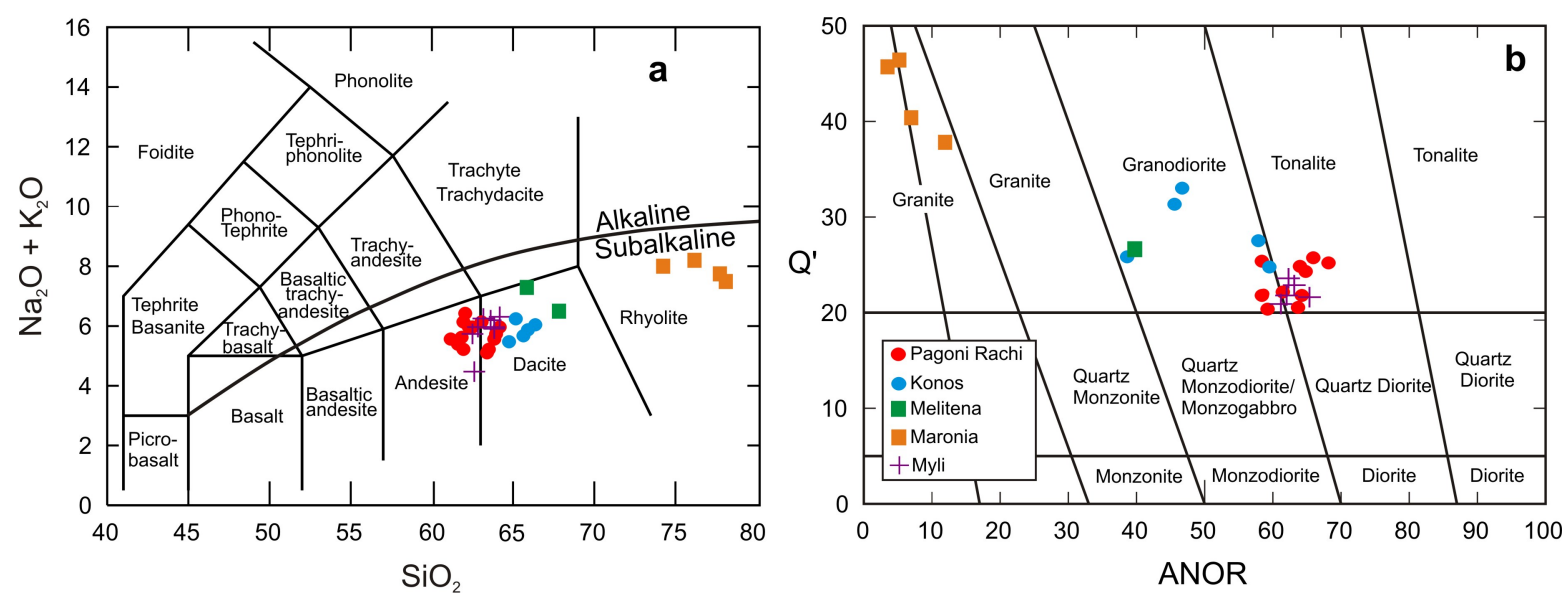


\subsection{Maronia}

In the Maronia area (about $10 \mathrm{~km}^{2}$ ), Mesozoic rocks of the Circum-Rhodope Belt were intruded by a Tertiary intrusive complex consisting of shoshonitic monzonite-gabbro [49]. This complex was subsequently intruded by a microgranite porphyry (Figure 3), hosting Re-rich porphyry $\mathrm{Cu}-\mathrm{Mo}-\mathrm{Au}$ mineralization $[39,49]$. The microgranite porphyry comprises phenocrysts of quartz, K-feldspar, biotite and minor plagioclase in a fine-grained matrix of quartz, K-feldspar and plagioclase. Apatite, zircon, amphibole, titanite and rutile are accessory minerals [39].

\subsection{Melitena}

The Melitena area (about $16 \mathrm{~km}^{2}$ ) contains Tertiary calc-alkaline and shoshonitic sub-volcanic to volcanic rocks (high-K calc-alkaline andesites to dacites, shoshonitic andesites, trachytic lavas, rhyodacitic ignimbrites and rhyolites; [53]), which were emplaced along E-W trending graben structures within metamorphic rocks (gneisses, amphibolites and marbles) of the Rhodope Massif (Figure 2). The porphyry $\mathrm{Mo}-\mathrm{Cu}-\mathrm{Au}$ mineralization is hosted within a subvolcanic granodiorite porphyry (Figure 3) characterized by phenocrysts of K-feldspar, plagioclase, biotite and hornblende. Quartz, magnetite and titanite are present in the rock matrix. High-sulfidation epithermal style alteration and mineralization extend over an area of about $20 \mathrm{~km}^{2}$ to the west of the Melitena porphyry prospect [54].

\section{Mineralization and Alteration}

The alteration assemblages and vein types recognized on the basis of surface relationships are distinguished according to the terminology of Gustafson and Hunt [55], Dilles and Einaudi [56], Muntean and Einaudi [57] for A, B, C and D veins and summarized by Seedorff et al. [58]. According to the above classification schemes, A-type veins formed early and consist mostly of quartz, K-feldspar, and sulfide ( \pm anhydrite) that have well-developed K-feldspar alteration generally within the porphyry stock. B-type veins formed after A-type veins along the margins of the stock and into the country rock. They are composed of quartz and sulfides including molybdenite ( \pm anhydrite) and generally lack an alteration halo. C-type veins contain quartz \pm chlorite \pm biotite \pm epidote \pm chalcopyrite \pm bornite \pm pyrite \pm molybdenite, while D-type veins usually form very late in the paragenetic sequence of vein formation and are structurally controlled. The sulfide-to-quartz ratio is generally higher than in A- and B-type veins, with banded anhydrite-sulfide textures being common. Sericite replaced K-feldspar and biotite in alteration haloes surrounding veins. Veins dominated by magnetite, actinolite and plagioclase are spatially associated with sodic or calcic alteration in some tonalitic-granodioritic porphyry $\mathrm{Cu}-(\mathrm{Au}-\mathrm{Mo})$ deposits, such as Park Premier, Utah and Island Copper, British Columbia and were termed $\mathrm{M}$ veins by [59]. However, magnetite veins can also be related to potassic alteration and range from magnetite-rich veins with biotite and minor quartz, chalcopyrite and K-feldspar envelopes, to sugary quartz veinlets with magnetite and chalcopyrite and no alteration envelopes. Such veinlets were termed A veinlets by [57,58]. 


\subsection{Sapes-Kirki-Esymi}

The recently discovered porphyry-style mineralization at Konos (Sapes) with a surface exposure of about $3 \mathrm{~km} \times 2 \mathrm{~km}$ is characterized by disseminated pyrite and chalcopyrite in sericitically altered granodiorite-tonalite porphyry host rocks, which are crosscut by dark-grey banded quartz veins, banded quartz veins (B-veins, using the terminology of [55]), molybdenite-quartz-pyrite veins, and D-type sulfide-rich gypsum veins [46-48] (Figure 4a,b). Fluid inclusion studies show that the porphyry-type quartz veins formed at around $500{ }^{\circ} \mathrm{C}$ from boiling fluids $[47,48]$. Molybdenite is associated with pyrite and rheniite (Figure $5 \mathrm{a}-\mathrm{d}$ ).

At Pagoni Rachi (Kirki), early porphyry-style mineralization (surface exposure of quartz stockworks is about $1 \mathrm{~km} \times 1 \mathrm{~km}$ ) consists of sulfides (pyrite, chalcopyrite, bornite, pyrrhotite, Re-rich molybdenite), iron oxides (magnetite and hematite) and native gold as disseminations, fracture fillings, and quartz veinlets, spatially related to a central $\mathrm{Na}-\mathrm{K}-\mathrm{Ca}-$-silicate alteration zone (albite/K-feldspar, biotite, actinolite, fluorite and magnetite). Molybdenum was mainly introduced as chalcopyrite-molybdenite-rheniite-pyrite-bearing quartz stockworks and as fissure coatings within late albite-chlorite-, albite-sericite- and sericite-carbonate altered granodiorite-tonalite porphyry (Figure 4c,d), similar to the Konos porphyry system. Native gold also occurs as grains up to $100 \mu \mathrm{m}$ in size in pyrite in fissures in D-type veins. Molybdenite is intergrown with pyrite (Figure 5e) and rheniite (Figure 5f). Bulk ore analyses of porphyry-style ore at Pagoni Rachi show high concentrations of $\mathrm{Au}$ (up to $5.1 \mathrm{~g} / \mathrm{t}$, average $=0.57 \mathrm{~g} / \mathrm{t}$ ), up to $1 \mathrm{wt} \% \mathrm{Cu}$, up to $2000 \mathrm{~g} / \mathrm{t} \mathrm{Mo} \mathrm{(average} \mathrm{Cu} / \mathrm{Mo}=22$ ), up to $20 \mathrm{~g} / \mathrm{t} \mathrm{Re}$, and up to $0.03 \mathrm{ppb}$ Pd [15] (Table 1).

The Myli Cu-Mo prospect (Esymi) is characterized by molybdenite-pyrite-bearing quartz stockworks (surface exposure of about $0.5 \mathrm{~km} \times 1 \mathrm{~km}$ ) that are similar to those spatially associated with Pagoni Rachi and Konos and to sericite- and sericite-carbonate alteration of the host granodiorite-tonalite (Figure 4e). Molybdenite and pyrite deposition formed close to the arsenopyrite/tennantite boundary (Figure $5 \mathrm{~g}-\mathrm{i}$ ). Native gold associated with chalcopyrite is enclosed in pyrite (Figure $5 \mathrm{j}$ ). This is, to our knowledge, only the second occurrence of native gold in porphyry-style stockworks in northeastern Greece other than that in sodic-potassic- and phyllic-altered rocks at Pagoni Rachi. Sericite, calcite-dolomite, and late kaolinite are gangue minerals within the veins (Figure $5 \mathrm{~g}, \mathrm{~h}$ ). Grades reach values up to $2100 \mathrm{~g} / \mathrm{t}$ $\mathrm{Cu}$, up to $210 \mathrm{~g} / \mathrm{t} \mathrm{Mo} \mathrm{(average} \mathrm{Cu} / \mathrm{Mo}=\sim 35$ ), and up to $0.2 \mathrm{~g} / \mathrm{t} \mathrm{Au}([60]$, Table 1 ).

\subsection{Maronia}

The Maronia porphyry $\mathrm{Cu}-\mathrm{Mo}-\mathrm{Au}$ deposit occurs in quartz stockworks (surface exposure of about $0.5 \mathrm{~km} \times 0.5 \mathrm{~km}$ ) crosscutting sericitic-altered microgranite (Figure 4f,g). Molybdenite is associated with pyrite, chalcopyrite, base metal sulfides, and sulfosalts [39]. According to [39], bulk ore analyses from porphyry-style ore have an average grade of $0.1 \mathrm{~g} / \mathrm{t} \mathrm{Au}$, and up to $2763 \mathrm{~g} / \mathrm{t} \mathrm{Cu}$ and $3909 \mathrm{~g} / \mathrm{t} \mathrm{Mo}$, with an average $\mathrm{Cu} / \mathrm{Mo}$ ratio of about 15 (Table 1 ).

\subsection{Melitena}

At the Melitena deposit, molybdenite forms disseminations and fissure fillings, and is associated with quartz veinlets crosscutting transitional sericitic-advanced argillic alteration zone of the granodiorite 
porphyry [54] (Figure 4h,i). Pyrite and molybdenite are the main metallic minerals (Figure 5k,1), whereas galena, chalcopyrite and pyrrhotite occur in minor amounts in the veins and as disseminations in the wallrocks. Bulk rock chemical analyses [17] reveal a high base and precious metal content $(\sim 400 \mathrm{~g} / \mathrm{t} \mathrm{Cu}, \sim 6000 \mathrm{~g} / \mathrm{t} \mathrm{Mo} \mathrm{(average} \mathrm{Cu} / \mathrm{Mo}=0.2)$, and up to $0.3 \mathrm{~g} / \mathrm{t} \mathrm{Au}($ average $=0.16 \mathrm{~g} / \mathrm{t})($ Table 1).

Figure 4. (a) Panoramic view of the Konos porphyry prospect, Sapes (from south to north). On top (Konos Hill) advanced argillic alteration, below sericitic alteration with quartz stockworks with molybdenite mineralization; (b) Surface outcrop of quartz + pyrite + molybdenite $(\mathrm{Qz}+\mathrm{Py}+\mathrm{Mol})$ veinlets overprinted by sericitic alteration of the granodiorite-tonalite porphyry at Konos; (c) Quartz + pyrite + chalcopyrite + molybdenite veinlets related to transitional sodic-potassic-sericitic alteration crosscut and reopened by pyrite-molybdenite $(\mathrm{Py}+\mathrm{Mol})$ veins (D-type) and associated sericitic alteration (Pagoni Rachi); (d) Molybdenite in sericitic altered granodiorite-tonalite porphyry at Pagoni Rachi; (e) Discontinuous, sinuous, wavy veins with irregular but sharply defined walls (A-type) re-opened by pyrite-molybdenite $(\mathrm{Py}+\mathrm{Mol})$ veins (D-type) and associated sericitic alteration at Myli; (f) View (from west to east) of the Maronia deposit, showing an outcrop of microgranite porphyry; (g) Quartz + pyrite + chalcopyrite + molybdenite $(\mathrm{Py}+\mathrm{Ccp}+\mathrm{Mol})$ veinlets crosscutting sericitic-altered microgranite porphyry at Maronia deposit; (h) View (from southwest to northeast) of the Melitena prospect. On top is advanced argillic alteration, whereas below sericitic alteration associated with quartz stockworks and molybdenite mineralization; and (i) Quartz + molybdenite (Mol) veinlets related to transitional sericitic-advanced argillic alteration of granodiorite porphyry at Melitena.
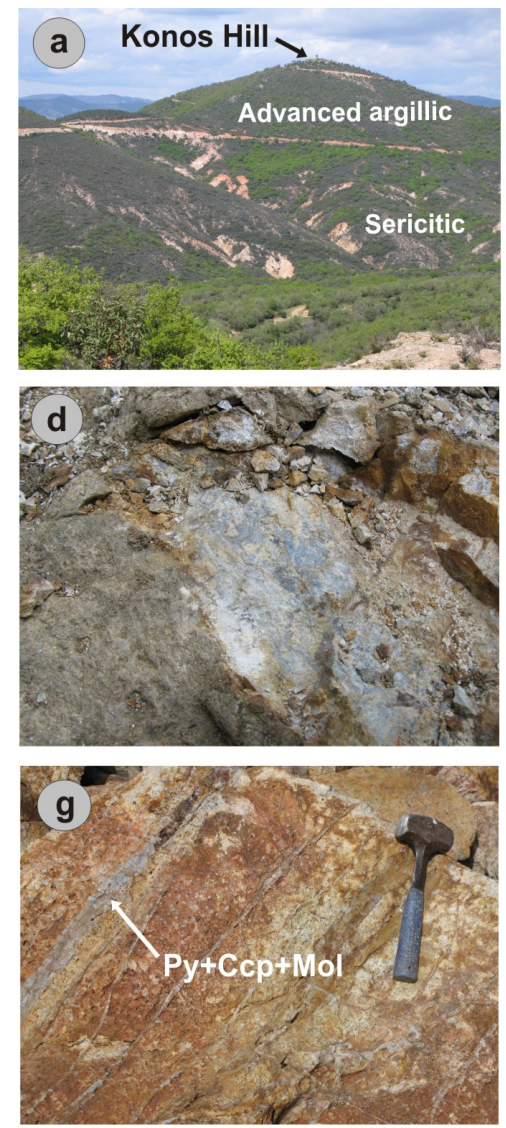
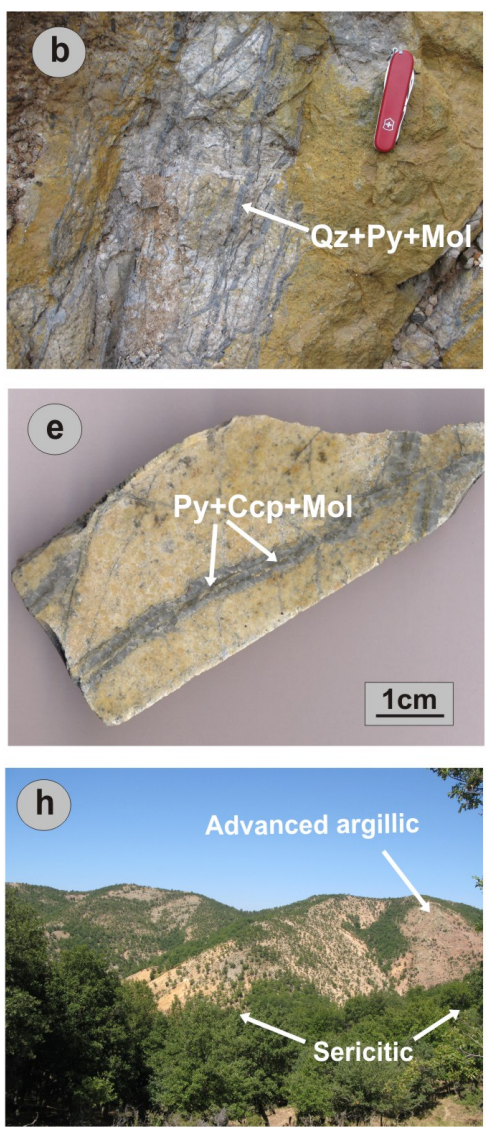
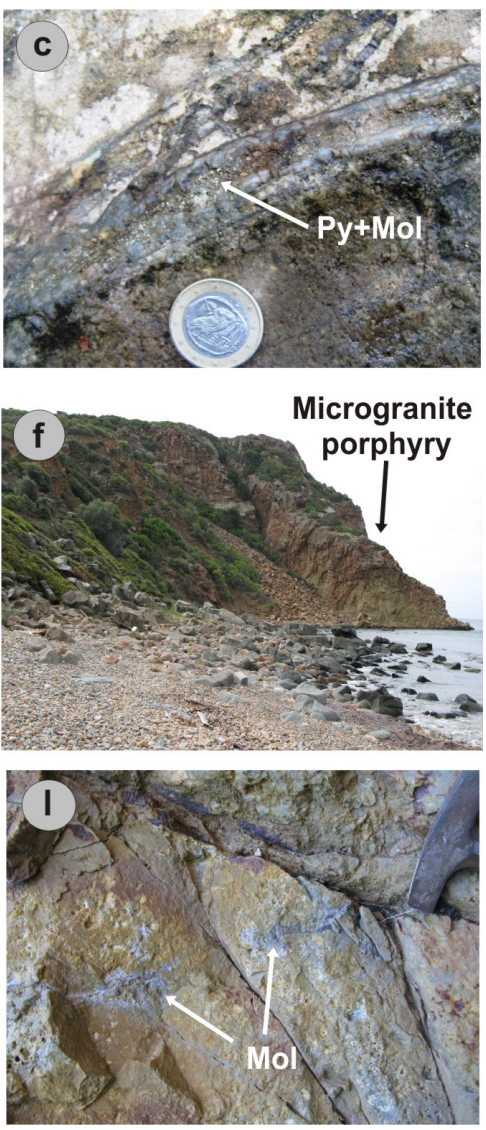
Figure 5. Photomicrographs of molybdenite and rheniite-bearing assemblages in quartz veins from the northeastern Greek prospects (a) Re-bearing molybdenite (Mol) with up to 1.9 wt \% Re, associated with pyrite (Py) and included in quartz (Qz) (SEM-BSE image, Konos); (b,c) Re-bearing molybdenite (Mol) surrounded by rheniite (Rhn). Pyrite (Py) and quartz (Qz) are also present (SEM-BSE images, Konos); (d) Zoned molybdenite crystal (Mol) with Re-content ranging from $0.65 \mathrm{wt} \% \mathrm{Re}$ in the core to $3.11 \mathrm{wt} \%$ towards the margin of the grain (SEM-BSE image, Konos); (e) Re-rich molybdenite (Mol) with pyrite (Py) and included in quartz (Qz) (reflected light, Pagoni Rachi); (f) Re-bearing molybdenite (Mol) interfingered and surrounded by rheniite (Rhn) (SEM-BSE image, Pagoni Rachi); (g) Molybdenite (Mol), pyrite (Py), arsenopyrite (Apy), accompanied by rutile (Rt), monazite (Mz), calcite (Cal), and quartz (Qz) (SEM-BSE image, Myli); (h) Molybdenite (Mol) and tennantite (Tnt) within quartz (Qz), sericite (Ser), kaolinite (Kao). Rutile (Rt) is also present (SEM-BSE image, Myli); (i) The Re content of the molybdenite crystal shown in Figure 5h (SEM-BSE image, Myli); (j) Native gold ( $\mathrm{Au}$ ) and chalcopyrite (Cpy) included in pyrite (Py) (SEM-BSE image, Myli); (k,l) Molybdenite (Mol) surrounding pyrite (Py) and associated quartz (Qz), sericite (Ser) (SEM-BSE image, Melitena).
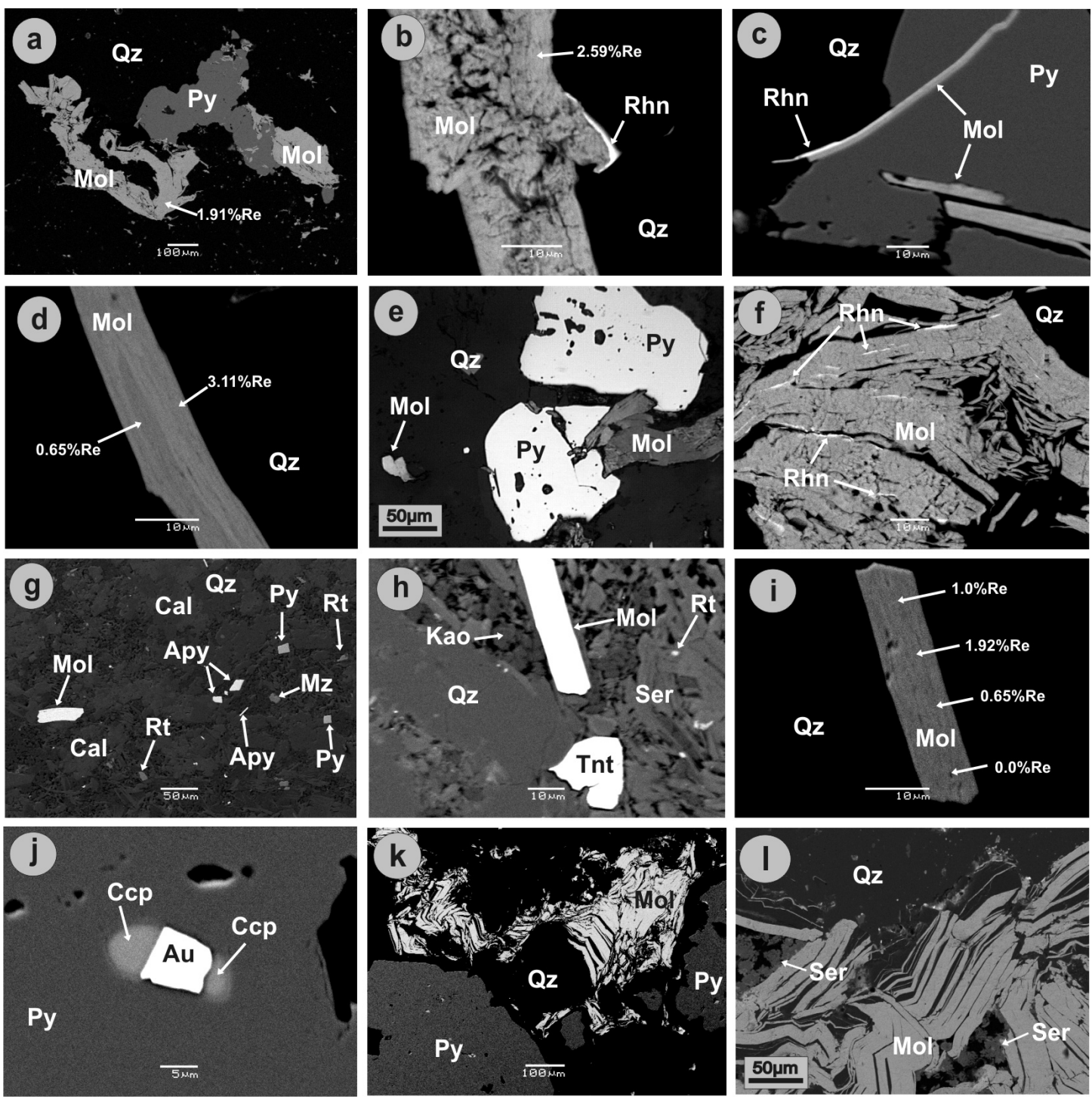
Table 1. Rhenium content of molybdenite from porphyry-type deposits in Greece and elsewhere. $\mathrm{Cu} / \mathrm{Mo}$ ratios and $\mathrm{Au}$ contents are also included.

\begin{tabular}{|c|c|c|c|c|c|c|c|c|}
\hline \multirow{2}{*}{ Deposit } & \multirow{2}{*}{ Type } & \multicolumn{4}{|c|}{ Re in molybdenites $(\mathrm{g} / \mathrm{t})$} & \multirow{2}{*}{$\mathrm{Cu} / \mathrm{Mo}$} & \multirow{2}{*}{$A u(g / t)$} & \multirow{2}{*}{ References } \\
\hline & & $n$ & Min. & Max. & Average & & & \\
\hline \multicolumn{9}{|c|}{ Greece } \\
\hline \multicolumn{9}{|l|}{$\underline{T H R A C E}$} \\
\hline Pagoni Rachi & Porphyry $\mathrm{Cu}-\mathrm{Mo}$ & 175 & 379 & 46,900 & 16,318 & 22 & 0.57 & [13-15] \\
\hline Konos & Porphyry $\mathrm{Cu}-\mathrm{Mo}$ & 7 & 750 & 31,100 & 15,621 & 4.95 & 0.04 & [15], this study \\
\hline Maronia & Porphyry $\mathrm{Cu}-\mathrm{Mo}$ & 55 & 1,200 & 28,800 & 7,600 & 15 & 0.10 & [39] \\
\hline Melitena & Porphyry $\mathrm{Mo} \pm \mathrm{Cu}$ & 49 & 2,100 & 17,400 & 7,900 & 0.2 & 0.16 & {$[15,17]$, this study } \\
\hline Myli & Porphyry $\mathrm{Cu}-\mathrm{Mo}$ & 32 & 440 & 19,200 & 2,733 & 35 & 0.09 & {$[15,60]$, this study } \\
\hline Kimmeria & Porphyry Mo-W & 47 & 10 & 550 & 134 & - & - & [15], this study \\
\hline \multicolumn{9}{|l|}{$\underline{\text { CHALKIDIKI }}$} \\
\hline Skouries & Porphyry $\mathrm{Cu}-\mathrm{Au}$ & 4 & 800 & 1,000 & 900 & 37 & 0.80 & [19] \\
\hline \multicolumn{9}{|l|}{$\underline{K I L K I S}$} \\
\hline Axioupolis & Porphyry Mo-W & 9 & 10 & 1,000 & 344 & - & - & {$[15]$, this study } \\
\hline \multicolumn{9}{|l|}{ AEGEAN SEA } \\
\hline Sardes, Limnos Isl. & Porphyry $\mathrm{Cu}-\mathrm{Mo}$ & 7 & 1,100 & 5,200 & 3,785 & - & - & {$[15,61]$} \\
\hline Fakos, Limnos Isl & Porphyry $\mathrm{Cu}$ & 5 & 910 & 2,220 & 1,396 & 69 & 0.03 & {$[15,62]$} \\
\hline Stypsi, Lesvos Isl. & Porphyry $\mathrm{Cu}$ & 10 & 300 & 10,600 & 2,460 & 40 & 0.10 & {$[15,61]$} \\
\hline Serifos & Porphyry Mo-W & 11 & 10 & 1,030 & 345 & - & - & [15], this study \\
\hline \multicolumn{9}{|l|}{ ATTICA } \\
\hline Lavrion & Porphyry Mo-W & 27 & 10 & 1,310 & 229 & - & - & [15], this study \\
\hline \multicolumn{9}{|c|}{ Bulgaria } \\
\hline Assarel & Porphyry Cu & 1 & - & - & 739 & 200 & 0.20 & {$[3,63-66]$} \\
\hline Elatsite & Porphyry $\mathrm{Cu}-\mathrm{Au}$ & 19 & 273 & 2,740 & 1,250 & $30-60$ & 0.21 & {$[19,63-67]$} \\
\hline Medet & Porphyry $\mathrm{Cu}$ & 22 & 565 & 1,163 & 905 & 37 & 0.10 & {$[3,63-67]$} \\
\hline \multicolumn{9}{|c|}{ Serbia } \\
\hline Bor & Porphyry $\mathrm{Cu}-\mathrm{Au}$ & 3 & - & - & 1,520 & - & 0.84 & {$[3,19,68]$} \\
\hline Majdnapek & Porphyry $\mathrm{Cu}-\mathrm{Au}$ & 3 & 2,320 & 3,550 & 2,770 & 120 & 0.35 & {$[68,69]$} \\
\hline \multicolumn{9}{|c|}{ Sweden } \\
\hline Aitik & Porphyry $\mathrm{Cu}$ & 13 & 20 & 784 & 226 & 133 & 0.2 & {$[70,71]$} \\
\hline \multicolumn{9}{|c|}{ Russia } \\
\hline Aksug & Porphyry $\mathrm{Cu}-\mathrm{Mo}$ & 1 & - & - & 460 & 70 & 0.06 & {$[19,72]$} \\
\hline Zhireken & Porphyry $\mathrm{Mo}-\mathrm{Cu}$ & 7 & 12 & 57 & 29 & 1 & 0.03 & {$[19,72]$} \\
\hline Sora & Porphyry $\mathrm{Mo}-\mathrm{Cu}$ & 9 & 6 & 18 & 14 & 15 & 0.02 & {$[19,72]$} \\
\hline Tominskoe & Porphyry $\mathrm{Cu}$ & & - & - & 1,080 & 113 & 0.12 & {$[3]$} \\
\hline \multicolumn{9}{|c|}{ Kazakhstan } \\
\hline Borly & Porphyry $\mathrm{Cu}-\mathrm{Au}$ & 19 & 250 & 5,500 & 3,160 & 31 & 0.3 & {$[19,73]$} \\
\hline Boshchekul & Porphyry $\mathrm{Cu}-\mathrm{Au}$ & 23 & 230 & 1,500 & 825 & 66 & 0.05 & {$[19,73]$} \\
\hline Kounrad & Porphyry $\mathrm{Cu}-\mathrm{Au}$ & 20 & 620 & 4,050 & 1,540 & 40 & 0.19 & {$[19,73]$} \\
\hline \multicolumn{9}{|c|}{ Uzbekistan } \\
\hline Kal'makyr & Porphyry $\mathrm{Cu}-\mathrm{Au}$ & 20 & 700 & 2,000 & 1,500 & 80 & 0.51 & {$[19,73]$} \\
\hline \multicolumn{9}{|c|}{ Armenia } \\
\hline Agarak & Porphyry $\mathrm{Cu}-\mathrm{Au}$ & 106 & 57 & 6,310 & 820 & 22 & 0.6 & {$[19,73]$} \\
\hline Kadzharan & Porphyry $\mathrm{Cu}-\mathrm{Au}$ & 237 & 33 & 2,620 & 245 & 13 & 0.65 & {$[19,73]$} \\
\hline
\end{tabular}


Table 1. Cont.

\begin{tabular}{|c|c|c|c|c|c|c|c|c|}
\hline \multirow{2}{*}{ Deposit } & \multirow{2}{*}{ Type } & \multicolumn{4}{|c|}{ Re in molybdenites $(\mathrm{g} / \mathrm{t})$} & \multirow{2}{*}{$\mathbf{C u} / \mathbf{M o}$} & \multirow{2}{*}{$A u(g / t)$} & \multirow{2}{*}{ References } \\
\hline & & $n$ & Min. & Max. & Average & & & \\
\hline \multicolumn{9}{|c|}{ Iran } \\
\hline Sar Cheshmeh & Porphyry $\mathrm{Cu}-\mathrm{Au}$ & 15 & 11 & 517 & 192 & 40 & 0.27 & [74] \\
\hline \multicolumn{9}{|c|}{ Mongolia } \\
\hline Erdenetium-Obo & Porphyry $\mathrm{Cu}-\mathrm{Mo}$ & 3 & 104 & 199 & 164 & 213 & 0.02 & {$[19,72]$} \\
\hline \multicolumn{9}{|c|}{ USA } \\
\hline Bagdad, Arizona & Porphyry $\mathrm{Cu}-\mathrm{Mo}$ & 9 & 330 & 642 & 479 & 40 & 0.001 & {$[19,73,75]$} \\
\hline Bingham, Utah & Porphyry $\mathrm{Cu}-\mathrm{Au}$ & 6 & 130 & 2,000 & 360 & 18 & 0.38 & {$[19,73,75]$} \\
\hline Castle Dome & Porphyry $\mathrm{Cu}$ & & 1,200 & 1,750 & 1,550 & 60 & 0.03 & {$[19,73,75]$} \\
\hline Esperanza & Porphyry $\mathrm{Cu}-\mathrm{Mo}$ & & 90 & 1,800 & 610 & 9 & 0.03 & {$[19,73,75]$} \\
\hline Miami & Porphyry $\mathrm{Cu}-\mathrm{Mo}$ & & & & 600 & 63 & 0.01 & {$[19,73,75]$} \\
\hline Morenci & Porphyry $\mathrm{Cu}-\mathrm{Mo}$ & 5 & 100 & 4,100 & 1,180 & 6 & 0,03 & {$[19,73,75]$} \\
\hline San Manuel & Porphyry $\mathrm{Cu}-\mathrm{Mo}$ & 5 & 700 & 1,200 & 950 & 55 & 0.02 & {$[19,73,75]$} \\
\hline Santa Rita & Porphyry $\mathrm{Cu}$ & 5 & 200 & 1,100 & 750 & 59 & 0.06 & {$[19,73,75]$} \\
\hline Silver Bell & Porphyry $\mathrm{Cu}-\mathrm{Mo}$ & 18 & 470 & 340 & 470 & 40 & 0.03 & {$[19,73,75]$} \\
\hline Twin Buttes & Porphyry $\mathrm{Cu}-\mathrm{Mo}$ & & & & 600 & 22 & 0.02 & {$[19,73,75]$} \\
\hline Climax & Porphyry Mo & 28 & 35 & 11 & 80 & & & {$[19,73,75]$} \\
\hline \multicolumn{9}{|c|}{ Canada } \\
\hline Adanac & Porphyry Mo & 4 & 8 & 22 & 12 & 0.02 & - & [3] \\
\hline Ajax West & Porphyry $\mathrm{Cu}-\mathrm{Au}$ & 1 & - & - & 3,161 & 62 & 0.2 & [3] \\
\hline Berg & Porphyry $\mathrm{Cu}-\mathrm{Mo}$ & 4 & 67 & 215 & 152 & 13 & 0.06 & [3] \\
\hline Bethlehem & Porphyry $\mathrm{Cu}$ & 3 & 190 & 980 & 553 & 80 & 0.01 & [3] \\
\hline Boss Mountain & Porphyry Mo & 7 & 49 & 157 & 80 & & & [3] \\
\hline Brenda & Porphyry $\mathrm{Cu}-\mathrm{Mo}$ & 12 & 95 & 145 & 115 & 4 & 0.01 & [3] \\
\hline Bronson Slope & Porphyry $\mathrm{Cu}-\mathrm{Au}$ & 1 & & & 180 & 28 & 0.44 & [3] \\
\hline Endako 1 & Porphyry Mo & 12 & 15 & 67 & 35 & 0.03 & - & [3] \\
\hline Endako 2 & Porphyry Mo & 3 & 204 & 397 & 302 & 0.03 & - & [3] \\
\hline Gibraltar & Porphyry $\mathrm{Cu}$ & 4 & 238 & 750 & 443 & 48 & 0.01 & [3] \\
\hline Glacier Gulch (Davidson) & Porphyry Mo & 2 & 34 & 41 & 38 & 0.23 & - & [3] \\
\hline Granisle & Porphyry $\mathrm{Cu}$ & 4 & 522 & 528 & 526 & 86 & 0.13 & {$[3]$} \\
\hline Huckleberry & Porphyry $\mathrm{Cu}$ & 2 & 247 & 258 & 253 & 86 & 0.13 & [3] \\
\hline Ingerbelle & Porphyry $\mathrm{Cu}-\mathrm{Au}$ & 1 & - & - & 1,620 & 215 & 0.16 & [3] \\
\hline Island Copper & Porphyry $\mathrm{Cu}$ & 2 & 1,704 & 1,863 & 1,784 & 38 & 0.19 & [3] \\
\hline Kemess South & Porphyry $\mathrm{Cu}-\mathrm{Au}$ & 2 & 3,106 & 4,609 & 3,858 & 28 & 0.65 & [3] \\
\hline Kitsault & Porphyry Mo & 2 & 57 & 102 & 80 & 0.03 & - & [3] \\
\hline Lomex & Porphyry $\mathrm{Cu}$ & 1 & - & - & 345 & 29 & 0.006 & [3] \\
\hline Maggie & Porphyry $\mathrm{Cu}-\mathrm{Mo}$ & 1 & - & - & 643 & 9.7 & - & [3] \\
\hline McIntyre-Copper Zone & Porphyry $\mathrm{Cu}-\mathrm{Au}$ & & & & 1,192 & 50 & 1.30 & [3] \\
\hline McLeod Lake & Porphyry $\mathrm{Cu}-\mathrm{Mo}$ & & & & 184 & 9 & 0.04 & [3] \\
\hline Mitchell (Sulphurets) & Porphyry $\mathrm{Cu}-\mathrm{Au}$ & 2 & 7,012 & 8,170 & 7,590 & 36 & 0.69 & [3] \\
\hline Ryan Lake & Porphyry $\mathrm{Cu}-\mathrm{Mo}$ & & & & 104 & 9 & 0.09 & [3] \\
\hline Schaft Creek & Porphyry $\mathrm{Cu}-\mathrm{Mo}$ & 1 & & & 590 & 13.2 & 0.18 & {$[3]$} \\
\hline
\end{tabular}


Table 1. Cont.

\begin{tabular}{|c|c|c|c|c|c|c|c|c|}
\hline \multirow{2}{*}{ Deposit } & \multirow{2}{*}{ Type } & \multicolumn{4}{|c|}{ Re in molybdenites $(\mathrm{g} / \mathrm{t})$} & \multirow{2}{*}{ Cu/Mo } & \multirow{2}{*}{$\mathrm{Au}(\mathrm{g} / \mathrm{t})$} & \multirow{2}{*}{ References } \\
\hline & & $n$ & Min. & Max. & Average & & & \\
\hline \multicolumn{9}{|c|}{ Philippines } \\
\hline Sipalay & Porphyry $\mathrm{Cu}$ & 1 & - & - & 1,700 & 34 & 0.05 & [22] \\
\hline Santo Thomas II & Porphyry $\mathrm{Cu}-\mathrm{Au}$ & 6 & 700 & 15,400 & 10,183 & 33 & 0.64 & [76] \\
\hline \multicolumn{9}{|c|}{ Peru } \\
\hline Michiquillay, Cajamarca & Porphyry $\mathrm{Cu}$ & 8 & 127 & 736 & 494 & 50 & 0.16 & [77] \\
\hline Galeno, Cajamarca & Porphyry $\mathrm{Cu}$ & 1 & - & - & 810 & 47 & 0.11 & [77] \\
\hline \multicolumn{9}{|c|}{ Chile } \\
\hline Chuquicamata & Porphyry $\mathrm{Cu}-\mathrm{Mo}$ & 3 & 194 & 245 & 220 & 16 & 0.01 & {$[19,73]$} \\
\hline Collahuasi & Porphyry $\mathrm{Cu}-\mathrm{Mo}$ & 2 & 368 & 448 & 410 & 22 & 0.01 & {$[19,73]$} \\
\hline El Salvador & Porphyry $\mathrm{Cu}$ & & & & 570 & 141 & 0.01 & {$[19,73]$} \\
\hline El Teniente & Porphyry $\mathrm{Cu}$ & 6 & 182 & 1,154 & 390 & 48 & 0.01 & {$[19,73]$} \\
\hline Escondida & Porphyry $\mathrm{Cu}-\mathrm{Au}$ & 1 & - & - & 1,355 & 156 & 0.25 & {$[19,73]$} \\
\hline Los Pelambres & Porphyry $\mathrm{Cu}-\mathrm{Mo}$ & 3 & 450 & 820 & 600 & 39 & 0.03 & {$[19,73]$} \\
\hline
\end{tabular}

\section{Mineralogy of Rhenium-Bearing Phases}

The Re content of molybdenite from prospects and deposits in northeastern Greece are presented in Tables 1 and 2 and plotted in Figure 6. Table 1 also includes data from other Greek porphyry systems (e.g., Skouries, Fakos, Sardes, Stypsi) and from molybdenite in porphyry $\mathrm{Cu}, \mathrm{Cu}-\mathrm{Au}, \mathrm{Cu}-\mathrm{Mo}$ and $\mathrm{Mo}$ deposits elsewhere. The almost perfect linear correlation between the Mo and Re content of molybdenite (Figure 6b) indicates that Re substitutes for Mo in the structure of molybdenite as previously suggested [13,21-23]. This is also evident from the homogeneous Re distribution in molybdenite (Figure 7a-c). As shown in Figure 5d,i, the Re content of molybdenite can vary greatly within the same crystal.

Table 2. Representative electron microprobe analyses and atomic proportions of molybdenite from Konos (1,2), Myli $(3,4)$, Melitena $(5,6)$, Maronia $(7,8)$ and Pagoni Rachi $(9,10)$; bd: below detection.

\begin{tabular}{ccccccccccc}
\hline wt \% & $\mathbf{1}$ & $\mathbf{2}$ & $\mathbf{3}$ & $\mathbf{4}$ & $\mathbf{5}$ & $\mathbf{6}$ & $\mathbf{7}$ & $\mathbf{8}$ & $\mathbf{9}$ & $\mathbf{1 0}$ \\
\hline $\mathrm{Mo}$ & 55.11 & 55.59 & 60.06 & 57.25 & 58.76 & 58.90 & 59.09 & 57.57 & 56.82 & 57.44 \\
$\mathrm{Re}$ & 3.11 & 2.15 & 1.92 & 0.91 & 1.74 & 1.33 & 1.22 & 2.00 & 4.14 & 3.28 \\
$\mathrm{Fe}$ & $\mathrm{bd}$ & 0.57 & bd & bd & bd & 0.02 & bd & bd & bd & 0.51 \\
$\mathrm{~S}$ & 42.98 & 41.67 & 38.48 & 40.90 & 39.03 & 39.08 & 39.49 & 39.65 & 39.25 & 39.21 \\
Total & 101.20 & 99.98 & 100.46 & 100.32 & 99.54 & 99.33 & 99.76 & 99.23 & 100.21 & 100.44 \\
\hline \multicolumn{8}{c}{ Chemical formula (based on 3 apfu) } \\
$\mathrm{Mo}$ & 0.891 & 0.914 & 1.006 & 0.934 & 0.999 & 1.001 & 0.996 & 0.974 & 0.966 & 0.972 \\
$\mathrm{Re}$ & 0.026 & 0.019 & 0.016 & 0.008 & 0.015 & 0.012 & 0.011 & 0.017 & 0.036 & 0.029 \\
$\mathrm{Fe}$ & 0.000 & 0.016 & 0.000 & 0.000 & 0.000 & 0.001 & 0.000 & 0.000 & 0.00 & 0.015 \\
$\mathrm{~S}$ & 2.082 & 2.051 & 1.978 & 2.058 & 1.986 & 1.987 & 1.993 & 2.008 & 1.997 & 1.984 \\
\hline
\end{tabular}


Figure 6. Re content of molybdenite, rheniite and intermediate molybdenite-rheniite phases from northeastern Greece (modified after $[13,14]$ and new data). (b) is the inset shown in (a).
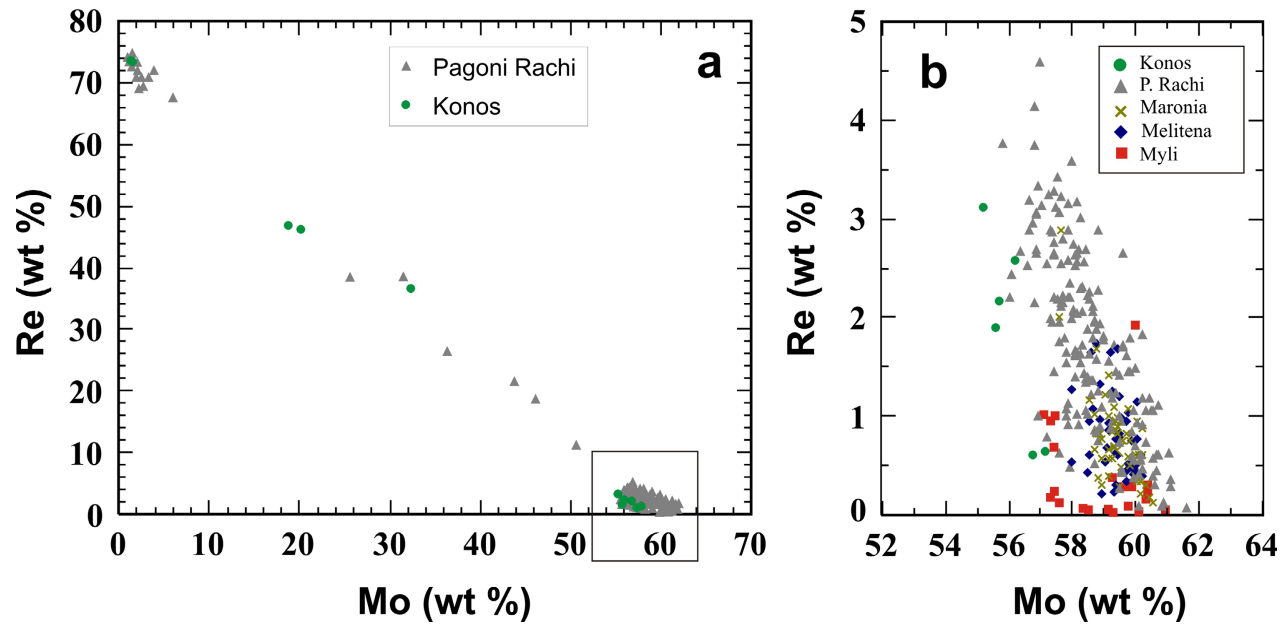

Figure 7. Back-scattered electron images showing the distribution of Mo, S and Re in molybdenite grains from Pagoni Rachi.
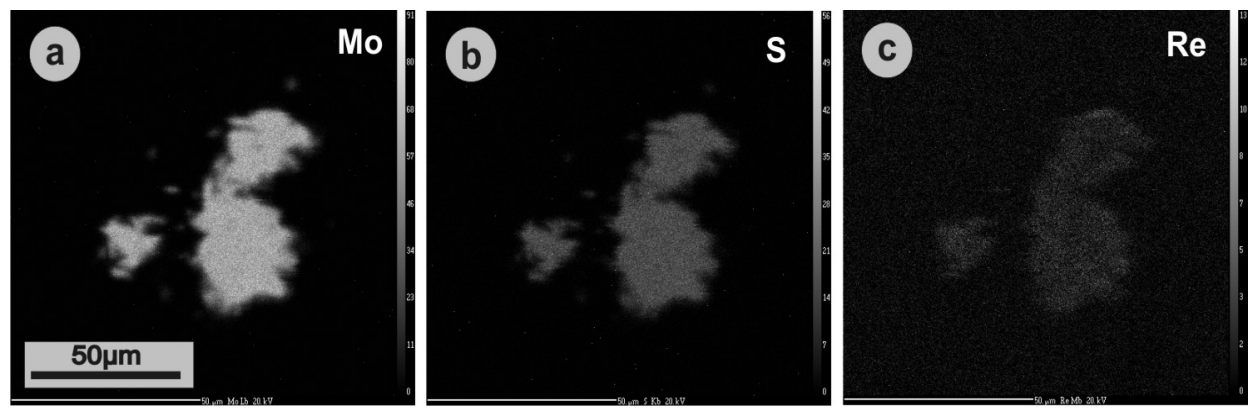

Experimental evidence presented by Drábek et al. [78], suggests that a maximum of $2.7 \mathrm{wt} \% \mathrm{Re}$ can be dissolved in molybdenite at temperatures up to $1000{ }^{\circ} \mathrm{C}$ (and $2.2 \mathrm{wt} \%$ at $400{ }^{\circ} \mathrm{C}$ ). Drábek et al. [78] cautioned that researchers studying molybdenite-bearing mineral assemblages containing anomalously high Re contents should "watch out for a possible excess Re phase." However, our structural analysis of four molybdenite crystals with the highest Re content ever reported in nature (up to $4.7 \mathrm{wt} \% \mathrm{Re}$ ) showed that Re actually enters the molybdenite structure as isovalent substituent of Mo. This observation is corroborated by the progressive shortening of the Mo-S mean bond distance, as well as by the uniform decrease of the unit-cell values with the increase of Re [13]. We conclude that the heterogeneity with up to $4.7 \mathrm{wt} \%$ Re in molybdenite is the result of solid solution, thus expanding the results of [78].

Pure rheniite and Mo-rich rheniite with up to $6 \mathrm{wt} \%$ Mo occurs at Pagoni Rachi and Konos porphyry systems ([13,14] and this study). It is believed that the Mo content of Mo-rich rheniite, may be derived from associated molybdenite. This is consistent with the experimental work of [78] that indicates a negligible amount of molybdenum in $\mathrm{ReS}_{2}$ at $400{ }^{\circ} \mathrm{C}$. Intermediate $(\mathrm{Mo}, \mathrm{Re}) \mathrm{S}_{2}$ and $(\mathrm{Re}, \mathrm{Mo}) \mathrm{S}_{2}$ phases with 10.5-38.5 wt \% Re at the Pagoni Rachi porphyry system [13], as well as intermediate phases between molybdenite and rheniite with 37.2-46.1 wt \% Re at the Konos porphyry prospect, may be due to the incorporation of rheniite in molybdenite and vice versa, as suggested by [78]. 
It was proposed by several workers that Re must have redistributed or decoupled within molybdenite after its formation [23,79-82]. These processes may explain the strong variation in the rhenium concentration within single grains. Rheniite, which rims molybdenite in the Pagoni Rachi and Konos porphyry deposits, resembles low temperature exsolution product of high-Re molybdenite. However, incorporation of the rheniite grains in quartz (Figure 5b,c) instead suggests that may have formed at the same time as molybdenite.

Among the second group of porphyries with a lower (but still high) content of Re in molybdenite (e.g., Skouries, Myli, Fakos, Sardes, Stypsi), the Myli prospect is hosted in granodiorite-tonalite porphyry, similar to that observed at Pagoni Rachi and Konos. The Re content of Myli molybdenite is lower (average $=2733 \mathrm{~g} / \mathrm{t}$ ). It should be noted here that other porphyry Mo and Mo-W systems in Greece are characterized by Re-free or very low-Re molybdenites (Re-content from 10 to $1310 \mathrm{~g} / \mathrm{t}$, average = $206 \mathrm{~g} / \mathrm{t}$ ) (e.g., the intrusion-related systems of Lavrion, Serifos, Axioulolis and Kimmeria) (Table 1).

\section{Discussion}

\subsection{Causes of Re-Enrichment in Molybdenite from Northern Greece}

In a previous discussion on the Re content of molybdenite from Greece, Voudouris et al. [14] proposed that Re-poor molybdenite is spatially associated mostly with porphyry Mo and Mo-W systems (e.g., Kimmeria, Lavrion, Serifos and Axioupolis deposits). This proposal is consistent with the findings of $[3,23,83,84]$ who suggested that the metal source of the deposit and the degree of magma fractionation are probable key factors that contribute to the Re content of molybdenite in granitoid-hosted deposits, and that the Re content of molybdenite decreases gradually from mantle sources, to mixtures between mantle and crust, and is the lowest in crustal rocks. It was suggested previously that primary magmatic compositional controls, such as those that influence ore element ratios including $\mathrm{Cu}: \mathrm{Mo}$ may also be an important control on the Re content of molybdenite [84]. According to Blevin [84], the atomic Re:Mo ratios of molybdenite and the degree of fractionation of the related granites have a strongly negative correlation. Molybdenite with low Re:Mo ratios are associated with Mo-dominant mineralizing systems, which are genetically related to more evolved granites, whereas the highest atomic Re:Mo ratio occurs in molybdenite in $\mathrm{Cu}-\mathrm{Au}$ deposits. Low ppb Re-bearing molybdenite is quite common in reduced intrusion-related systems like the Mactung (Canada) and Pogo (Alaska) deposits [85,86].

All porphyry Mo and Mo-W systems hosting Re-poor molybdenite in Greece are either of crustal origin, or they originated from the mantle but with a major contribution of these metals from the crust. The molybdenite-bearing Kimmeria granodiorite was considered to have formed by dehydration melting of the lower crust without any contribution from mantle components [87,88], whereas the Lavrion granodiorite is regarded to have a mantle origin with a contribution of a crustal felsic component $[89,90]$. The source materials for the leucocratic, S-type Axioupolis and Serifos granites are considered to also have a crustal anatectic origin [91,92]. The low Re-content of molybdenite in the Mo-W-enriched Lavrion, Kimmeria, Axioulopis and Serifos systems is consistent with the reducing nature of mineralizing fluids as proposed by the experimental work of [93], who suggested that reducing fluids containing sulfur have a lower capacity for transporting rhenium. Such fluids are, 
therefore, not favorable for the formation of rhenium-enriched sulfide deposits. To form rhenium deposits, oxidizing fluids must be operative at some stage(s) of ore formation.

Geochemical and stable isotope data suggest that the Oligocene to Miocene plutonic rocks in northern Greece and associated porphyry mineralization with ultrahigh- and high-Re molybdenite, are derived from sub-continental lithospheric mantle and/or the lower crust, with a minimal contribution from the crust $[88,94]$. More precisely: (a) a LILE- and LREE-enriched sub-continental mantle is the proposed magma source for the Pagoni Rachi and Konos granodiorite-tonalite [95]; (b) a lower crustal origin for the Maronia microgranite [49]; (c) a mantle source for the high-K calc-alkaline granodiorite porphyry at Stypsi [94]; (d) dehydration melting of enriched metabasaltic amphibolite at the base of crust, triggered by rising mantle melts from both the asthenosphere and the enriched subcontinental lithosphere for igneous rocks related to porphyry-style mineralization on Limnos island [96]); and (e) a magma genesis in the upper mantle and subsequent crustal contamination of the parental melts during emplacement of the Skouries deposit [97].

The Re- and/or Te-enriched porphyry $\mathrm{Cu}-\mathrm{Mo}-\mathrm{Au}$ deposits found in northeastern Greece (Pagoni Rachi, Konos, Maronia, Melitena) formed in a post-subduction, post-collisional environment, where partial melting of previously metasomatized mantle lithosphere triggered by local ascent of asthenospheric mantle could be the key to metal enrichment $[13,43]$. In post-subduction tectonic settings, similarly to that in northern Greece, arc-like magmas and related porphyry and epithermal ore deposits formed by remelting of previously subduction-modified upper plate lithosphere, and in particular the lower crustal amphibolitic cumulate roots of former arc magmatic complexes [98,99]. In the parental magmas of the Pagoni Rachi and Konos intrusives, Re and other metals (e.g., $\mathrm{Mo}, \mathrm{Cu}, \mathrm{Fe}$ ) may be derived from the breakdown of amphibole in amphibolites as suggested by [100] for the recently discovered Longmendian Mo deposit, China. A melting of mafic or ultramafic rocks, as well as involvement of mantle underplating or metasomatism in the source rocks, could also contribute to Re-enrichment in molybdenite as proposed elsewhere [23].

Arc magmatism may also be responsible for the enrichment in Re and possibly of other fluid-mobile elements such as $\mathrm{Te}, \mathrm{Pb}, \mathrm{As}, \mathrm{Sb}, \mathrm{Cu}, \mathrm{Au}$, and the Pd-group elements in the mantle wedge, probably due to fluids released from the subducted slab and associated sediments [99,101-103]. The enrichment of $\mathrm{Au}$ and Te occurs in several porphyry- and epithermal-style deposits associated with post-subduction magmatism in the Neo-Tethyan belt, including the Rosia Montana (Romania), Larga (Metaliferi Mountains) and Sacarimb, Golden Quadrilateral (Romania) [104-107], Skouries (Greece) [108], the Grasberg, Ok Tedi, Porgera, Lihir, and Emperor porphyry $\mathrm{Cu}-\mathrm{Au}$ and epithermal $\mathrm{Au}$ deposits in the southwest Pacific, as well as the Golden Sunlight Mo-Au (Montana), and Mount Milligan $\mathrm{Cu}-\mathrm{Au}$ porphyry deposits, British Columbia [99,109,110]. This gold-enrichment may reflect remelting of small amounts of residual sulfide left in the deep lithosphere by arc magmatism [98,99]. According to [107], exceptional endowment in $\mathrm{Te}-\mathrm{Au}$ epithermal deposits is common to provinces associated with extensional magmatism preceded by much older mantle metasomatism. It is suggested here that a strongly metasomatized mantle wedge which triggered magmatism in northeastern Greece, was responsible for the Re- and Te-enrichment in the Greek magmatic-hydrothermal systems. Similar scenarios, where a melt-metal source in fertile mantle and/or juvenile lower crust was responsible for the relatively high Re concentrations (up to $0.35 \mathrm{wt} \%$ ) in molybdenite from the Majdanpek (Serbia) and Elatsite (Bulgaria) Cu-Mo porphyry deposits, is supported by [111]. Rhenium is directly derived from 
partial melting of metasomatized mantle whereby Re is released during dehydration of the subducted Garzê-Litang oceanic crust to form molybdenite with up to $2434 \mathrm{ppm}$ Re at the Xuejiping deposit, China [112]. A release of Re from the asthenospheric mantle wedge by subduction-related fluids and melts similarly to that associated with the formation of Kudryavy volcano, Kurile islands [103] could also explain the enrichment of Re in magmatic systems in northern Greece.

Local physicochemical conditions (e.g., $\mathrm{fO}_{2}$, activity of $\mathrm{Cl}$, pressure, and temperature) affect the transportation, deposition and zoning of metals (including Re) in magmatic-hydrothermal systems [19] and likely control the Re content of molybdenite. However, the Re-enriched mineralization in northeastern Greece expands over an area of about $5400 \mathrm{~km}^{2}$ and this suggests that large-scale processes (e.g., anomalous concentration of Re in source areas is necessary to account for the $\mathrm{Re}$ enrichment) in the studied occurrences.

\subsection{Implications for Re-Au Exploration}

According to [3], molybdenite from porphyry $\mathrm{Cu}-\mathrm{Au}$ deposits in Canada have higher $\mathrm{Re}$ contents (up to $3858 \mathrm{ppm}$ Re for Kemess South and $8170 \mathrm{ppm}$ Re for Mitchell) than molybdenite from Canadian porphyry $\mathrm{Cu}$ deposits (up to 1863 ppm Re for Island Copper). In non-Canadian porphyry $\mathrm{Cu}$ deposits, the Re content of molybdenite can be more than 4000 ppm at Kounrad, Kazakhstan and Morenci, USA and up to 15,400 ppm at the Santo Thomas II porphyry $\mathrm{Cu}-\mathrm{Au}$ deposit, Philippines [3,76]). The Re content of molybdenite in Canadian porphyry $\mathrm{Cu}-\mathrm{Mo}$ deposits is generally lower than that in molybdenite from porphyry $\mathrm{Cu}$ and $\mathrm{Cu}-\mathrm{Au}$ deposits (e.g., up to $643 \mathrm{ppm}$ Re from the Maggie deposit; [3]). In non-Canadian $\mathrm{Cu}-\mathrm{Mo}$ deposits the Re content of molybdenite may reach values up to $2000 \mathrm{ppm}$ (e.g., Bingham, USA), whereas the Re content of molybdenite in porphyry Mo deposits is characterized by being much lower (up to 397 ppm, e.g., Endako) [3]. The Merlin Mo-Re deposit, Australia, is by far the highest grade Re deposit in the world with indicated mineral resources of $6.7 \mathrm{Mt}$ at $1.4 \%$ Mo and $23 \mathrm{~g} / \mathrm{t} \operatorname{Re}[24,25]$. However, despite being the highest grade Re deposit in the world, the Mo/Re ratio is about 725 [25] with an average Re content in molybdenite of about $1000 \mathrm{ppm}$. In general, the plot of $\mathrm{Re}$ content of molybdenite versus $\mathrm{Cu} / \mathrm{Mo}$ ratio and $\mathrm{Au}$ grade in porphyry-type deposits (Figure 8a,b) shows a correlation in which the Re content of the molybdenite increases as the $\mathrm{Cu} / \mathrm{Mo}$ ratio decreases (e.g., in porphyry $\mathrm{Cu}-\mathrm{Au}$ and $\mathrm{Cu}$ deposits [3]). These authors also demonstrated that platinum-group metals, and gold, are concentrated in porphyry deposits along with Re, and that high contents of Re in molybdenite can be an indicator of high levels of platinum-group metals.

Preliminary data on $\mathrm{Au}$ grades and $\mathrm{Cu} / \mathrm{Mo}$ ratios versus the $\mathrm{Re}$ content of molybdenite in the porphyry deposits in northeastern Greece (Figure 8a,b) suggest that molybdenite in these occurrences have characteristically high Re contents. The Pagoni Rachi prospect is highly anomalous in Au and ongoing exploration is needed to verify if the rest of the prospects are also enriched in Au and Re, since high Re content of molybdenite correlates with high Au grades in these deposit. However, since these occurrences are not associated with any measured resources, it is premature to consider them as a separate class or subclass of porphyry deposits. The Re-in-molybdenite values from the Skouries deposit (the only porphyry deposit in Greece with a significant resource) average $900 \mathrm{ppm}$ Re, which is low compared to other porphyry $\mathrm{Cu}-\mathrm{Au}$ deposits worldwide, but this maybe a function of the limited number of molybdenite analyses (i.e., four) obtained here. 
Figure 8. (a) Re content of molybdenite vs. Cu/Mo ratio of porphyry deposits; (b) $\mathrm{Re}$ content of molybdenite $v s$. gold grades of porphyry deposits (modified after [3]). Re content of molybdenite, $\mathrm{Cu} / \mathrm{Mo}$ ratio and $\mathrm{Au}$ grades of studied prospects are also shown.
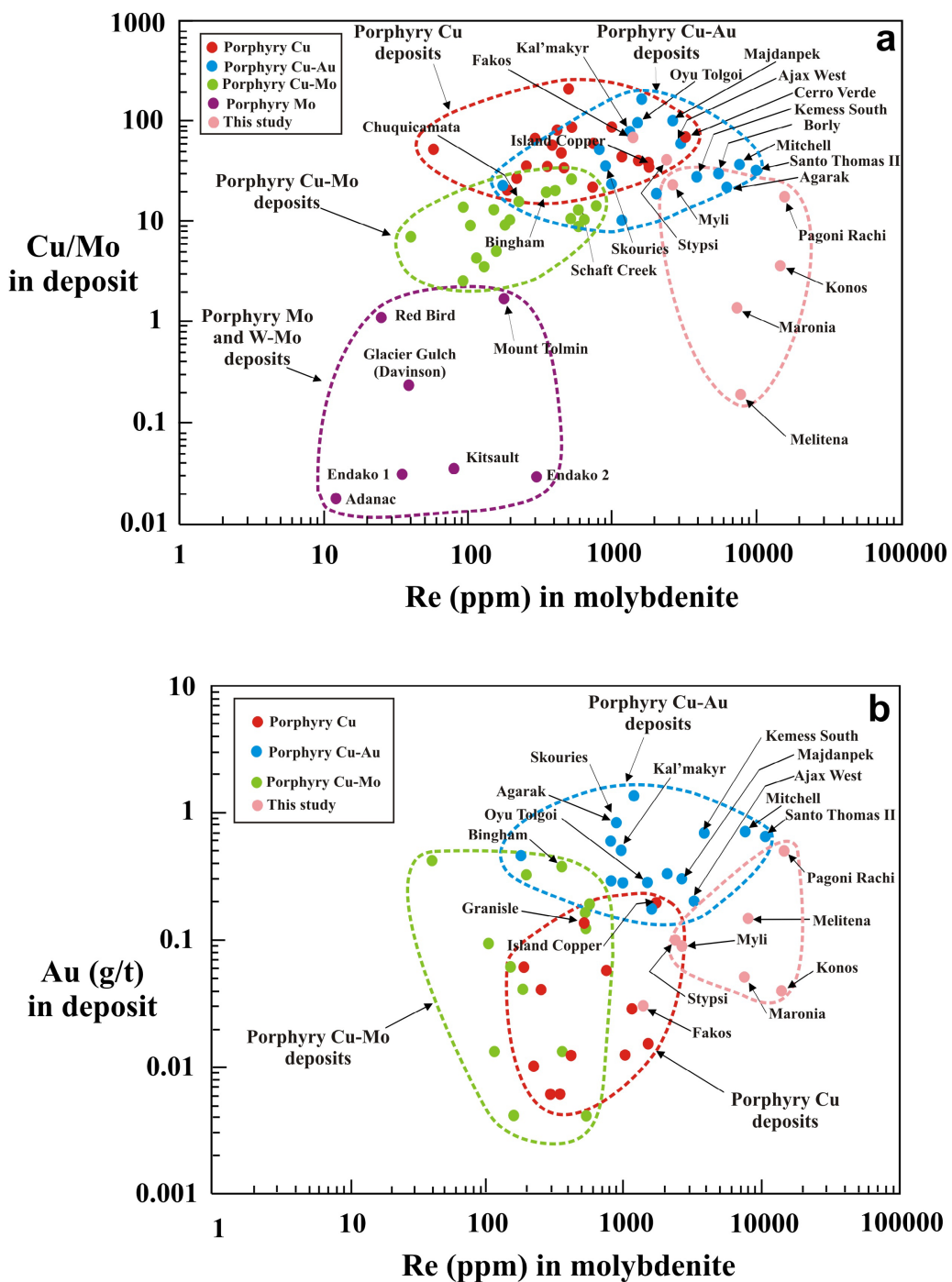

Our data suggest enrichment of Re in molybdenite throughout the studied deposits and at various levels of exposure in the system ([13] and this study): Although these deposits are characterized by a strong telescoping (where sericitic alteration overprints earlier sodic-potassic alteration and A, B-type quartz veinlets are reopened by D-type veinlets), molybdenite remains Re-enriched in all mineralized stages, as well as in both the core and distal parts of the systems. Based on available data, there is no indication that the Re values in molybdenite vary with depth and/or laterally in these occurrences, as appears to be the case at, for example, Bingham Canyon by [75], where highest values are present in the upper part of the deposit (up to $2000 \mathrm{ppm} \mathrm{Re}$ ) and decreased with depth (to less than $200 \mathrm{ppm}$ ).

The lower Re content of molybdenite from the Myli deposit may be attributed to the slightly reduced nature of the ore-bearing fluids (presence of arsenopyrite together with molybdenite) as opposed to highly oxidizing conditions at Pagoni Rachi and Konos systems. The reduced nature of the fluid may be due to the intrusion of granodiorite-tonalite porphyry in reduced sediments at Myli. This is also the case for the two porphyry systems on Limnos island (Fakos and Sardes) [14,61,62]. Stein et al. [23] and Gilles and Schilling [75] related the Mo contents to mass balance considerations in 
which the $\mathrm{Re}$ content of molybdenite in $\mathrm{Cu}-\mathrm{Mo}$ deposits was higher than for molybdenite in porphyry Mo deposits because of the lower amount of molybdenite in the former deposits. However, our study indicates that there is no clear relationship between Re content and molybdenite abundance, since molybdenite at the Maronia and Melitena porphyry $\mathrm{Mo}-\mathrm{Cu}$ deposits is a very abundant mineral that is extremely enriched in Re.

The diaspore + pyrophyllite + aluminum-phosphate-sulfate mineral-bearing root zones of high-temperature advanced argillic lithocaps in northern Greece may be potential exploration targets for porphyry type $\mathrm{Cu}-\mathrm{Mo}-\mathrm{Re}-\mathrm{Au}$ mineralization [54]. In the Greek Rhodope region, almost all high-sulfidation epithermal systems and associated advanced argillic alteration zones are telescoped onto porphyry-style $\mathrm{Cu}-\mathrm{Mo}-\mathrm{Re}-\mathrm{Au}$ mineralization (Figure 9). Careful mapping of advanced argillic alteration assemblages in the Rhodope Massif has the potential to identify new target areas for porphyry-style mineralization. Ongoing exploration and geological, mineralogical and geochemical studies will determine whether or not northeastern Greece represents a $\mathrm{Re}-\mathrm{Au}-\mathrm{Te}$ metallogenetic province.

Figure 9. Schematic diagram integrates magma emplacement, degassing and porphyry-epithermal mineralizing processes in northeastern Greece. Four successive magmatic pulses (granodiorite-tonalite porphyry, monzodiorite-monzogabbro, microdiorite and microgranite porphyry) within metamorphic basement and coeval volcanic rocks resulted in superimposed porphyry-epithermal style of mineralization and upgrading of earlier formed mineralization. Magmatic vapors resulted in the formation of high-sulfidation $\mathrm{Cu}-\mathrm{Au}-\mathrm{Te}$ mineralization within advanced argillic alteration lithocaps and fluid inclusion evidence suggests that rheniite, Re-rich molybdenite, chalcopyrite and native gold at Pagoni Rachi and Konos prospects may have precipitated as temperatures fell below $400{ }^{\circ} \mathrm{C}$ during phase separation at relatively oxidized conditions, high chlorine activity, and from relatively acid hydrothermal solutions.

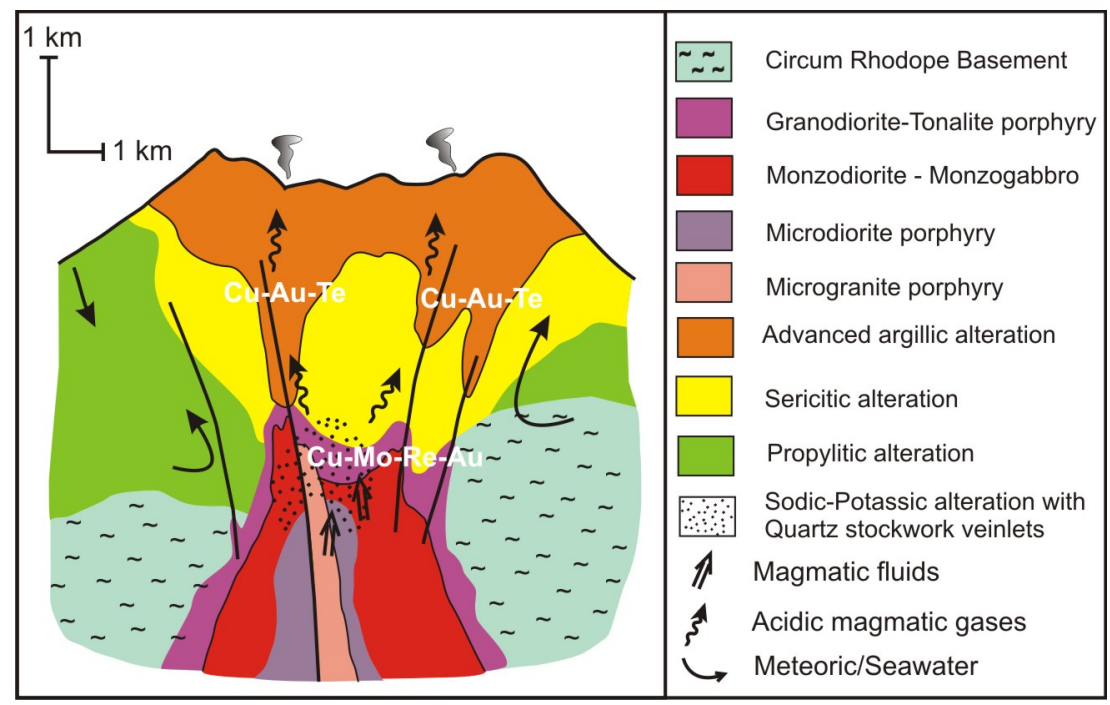

\section{Conclusions}

Molybdenite in Oligocene porphyry systems in northeastern Greece contain extremely high Re content (several tens of thousands of ppm) which is associated with pure rheniite, Mo-rich rheniite, and 
intermediate (Mo, Re) $\mathrm{S}_{2}$ and $(\mathrm{Re}, \mathrm{Mo}) \mathrm{S}_{2}$ phases with up to $46.1 \mathrm{wt} \% \mathrm{Re}$, the latter probably being the result of micro-inclusions of rheniite in molybdenite and vice versa. The molybdenite-rheniite mineralization is hosted by sodic-potassic, sodic-sericitic- and sericitic-altered granodiorite-tonalite and microgranite porphyries and is spatially associated with native gold, $\mathrm{Fe}-\mathrm{Cu}$ sulfides and oxides. Although local factors at deposit scale may have contributed to Re content in molybdenite, we favor large scale processes, based on the large areal distribution of Re-enrichment in magmatic-hydrothermal systems in northeastern Greece. Strong chemical inhomogenities in the mantle wedge which triggered post-subduction magmatism in northeastern Greece during the Tertiary, resulted in the Re- (and Te, $\mathrm{Au}$ ) enrichment in these porphyry systems. The widespread presence of precious metal tellurides in the ores, and first detection of Pd in Pagoni Rachi, further suggests that these systems could be exploration targets for porphyry-style $\mathrm{Au}-\mathrm{Re}-\mathrm{Pd}-\mathrm{Te}$ ores.

\section{Acknowledgments}

We thank S. Heidrich and Warren Straszheim for kindly providing access to and assistance with the electron microprobe at Hamburg and Iowa State Universities, respectively. E. Michailidis is especially thanked for his assistance with SEM-EDS analyses at Athens University. The reviewers are greatly acknowledged for their helpful and constructive comments.

\section{References}

1. Newberry, R.J.J. Polytypism in molybdenite (I): A non-equilibrium, impurity-induced phenomenon. Am. Mineral. 1979, 64, 758-767.

2. Melfos, V.; Voudouris, P.; Arikas, K.; Vavelidis, M. Rhenium-rich molybdenites in Thracian $\mathrm{Mo} \pm \mathrm{Cu}$ porphyry occurrences, NE Greece [in Greek]. Bull. Geol. Soc. Greece 2001, 34 1015-1022.

3. Sinclair, D.W.; Jonasson, I.R.; Kirkham, R.V.; Soregaroli, A.E. Rhenium and Other Platinum-Group Metals in Porphyry Deposits; Open File 6181; Geological Survey of Canada: Ottawa, Canada, 2009.

4. Ekström, M.; Hålenius, U. A new rhenium-rich sulfide from two Swedish localities. N. Jb. Miner. Mnh. 1982, 1, 6-10.

5. Mitchell, R.H.; Laflamme, J.H.; Cabri, L.J. Rhenium sulfide from the Coldwell Complex, northwester Ontario. Mineral. Mag. 1989, 53, 635-637.

6. Tarkian, M.; Housley, R.M.; Volborth, R.M.; Greis, O.; Moh, G. Unnamed Re-Mo-Cu sulfide from the Stillwater Complex, and crystal chemistry of its synthetic equivalent spinel type $(\mathrm{Cu}, \mathrm{Fe})(\mathrm{Re}, \mathrm{Mo})_{4} \mathrm{~S}_{8}$. Eur. J. Mineral. 1991, 3, 977-982.

7. Barkov, A.Y.; Lednev, A.I. A rhenium-molybenum-copper sulfide from the Lukkulaisvarra layered intrusion, northern Karelia, Russia. Eur. J. Mineral. 1993, 6, 1227-1233.

8. Kojonen, K.K.; Roberts, A.C.; Isomaki, O.P.; Knauff, V.V.; Johanson, B.; Pakkanen, L. Tarkianite, $(\mathrm{Cu}, \mathrm{Fe})(\mathrm{Re}, \mathrm{Mo})_{4} \mathrm{~S}_{8}$, a new mineral species from the Hitura mine, Nivala, Finland. Can. Mineral. 2004, 42, 539-544.

9. Bobrov, A.; Hurskiy, D.; Merkushyn, I.; Voloshyn, O.; Stepanyuk, L.; Lysenko, O.; Goshovksi, S. The First Occurrence of Native Rhenium in Natural Geological Systems. In Proceedings of 33rd International Geological Congress, Oslo, Norway, 6-14 August 2008. 
10. Poplavko, E.M.; Marchakova, I.D.; Zak, S.Sh. A rhenium mineral in the ores of the Dzhezkazgan locality [in Russian]. Dokl. Acad. Nauk. USSR 1962, 146, 433-436.

11. Lavrov, O.B.; Kuleshevich, L.V. The first finds of rhenium minerals in Karelia. Dokl. Earth Sci. 2010, 432, 598-601.

12. Korzhinsky, M.A.; Tkachenko, S.I.; Shumulovich, K.I.; Taran, Y.A.; Steinberg, G.S. Discovery of a pure rhenium mineral at Kudriavy volcano. Nature 1994, 369, 51-52.

13. Voudouris, P.C.; Melfos, V.; Spry, P.G.; Bindi, L.; Kartal, T.; Arikas, K.; Moritz, R.; Ortelli, M.; Rhenium-rich molybdenite and rheniite $\left(\mathrm{ReS}_{2}\right)$ in the Pagoni Rachi-Kirki Mo-Cu-Te-Ag-Au deposit, Northern Greece: Implications for the rhenium geochemistry of porphyry style $\mathrm{Cu}-\mathrm{Mo}$ and Mo mineralization. Can. Mineral. 2009, 47, 1013-1036.

14. Voudouris, P.; Melfos, V.; Moritz, R.; Spry, P.G.; Ortelli, M.; Kartal, T. Molybdenite Occurrences in Greece: Mineralogy, Geochemistry and Rhenium Content. In Scientific Annals of the School of Geology AUTH, Proceedings of the XIX Congress of the Carpathian-Balkan Geological Association, Thessaloniki, Greece, 23-26 September 2010; pp. 369-378.

15. Voudouris, P.C.; Melfos, V.; Spry, P.G.; Kartal, T.; Schleicher, H.; Moritz, R.; Ortelli, M. The Pagoni Rachi/Kirki $\mathrm{Cu}-\mathrm{Mo} \pm \mathrm{Re} \pm \mathrm{Au}$ deposit, northern Greece: Mineralogical and fluid inclusion constrains on the evolution of a telescoped porphyry-epithermal system. Can. Mineral. 2013, 51, 411-442.

16. Arikas, K. A porphyry Mo-Cu occurence near Kirki (western Thrace, Greece). N. Jb. Miner. Abh. 1979, 137, 74-82.

17. Filippidis, A.; Vavelidis, M.; Michailidis, K.; Evangelou, E. Re-rich and Re-poor molybdenite in the Melitena porphyritic intrusion, Rhodope massif. Fortschr. Mineral. 1986, 64, 47.

18. Michailidis, K.; Filippidis, A.; Kassoli-Fournaraki, A. Polytypism and rhenium-contents of molybdenites from two Mo-deposits in northern Greece. In Current Research in Geology Applied to Ore Deposits, Proceedings of the second biennial SGA meeting, Granada, Spain, 9-11 September 1993; Fenoll Hach-Ali, P., Torres-Ruiz, J., Gervilla, F., Eds; University of Granada: Granada, Spain, 1993; pp. 641-644.

19. Berzina, A.N.; Sotnikov, V.I.; Economou-Eliopoulos, M.; Eliopoulos, D.G. Distribution of rhenium in molybdenite from porphyry $\mathrm{Cu}-\mathrm{Mo}$ and $\mathrm{Mo}-\mathrm{Cu}$ deposits of Russia (Siberia) and Mongolia. Ore Geol. Rev. 2005, 26, 91-113.

20. Newberry, R.J.J. Polytypism in molybdenite (II): Relationships between polytypism, ore deposition/alteration stages and rhenium contents. Am. Mineral. 1979, 64, 768-775.

21. Fleisher, M. The geochemistry of rhenium with special reference to its occurrence in molybdenite. Econ. Geol. 1959, 54, 1406-1413.

22. Terada, K.; Osaki, S.; Ishihara, S.; Kiba, T. Distribution of rhenium in molybdenites from Japan. Geochem. J. 1971, 4, 123-141.

23. Stein, H.J.; Marke, R.J.; Morgan, J.W.; Hannah, J.L.; Scherstén, A. The remarkable Re-Os chronometer in molybdenite: how and why it works. Terra Nova 2001, 13, 479-486.

24. Brown, M.; Lazo, F.; Carter, P.; Goss, B.; Kirwin, D. The Geology and Discovery of the Merlin Mo-Re Zone of the Mount Dore Deposit, Mount Isa Inlier, NW Queensland, Australia. SGA News, 27 June 2010, pp. 9-15. 
25. Lycopodium Minerals QLD Pty Ltd. Merlin Molybdenum Rhenium Project: Feasibility Study Northwest Queensland, Australia; NI 43-101 Technical Report. Available online: http://www.ivanhoeaustralia.com/i/pdf/NI43-101_Merlin_Technical_Report.pdf (accessed on 16 April 2012).

26. Jolivet, L.; Brun, J.P. Cenozoic geodynamic evolution of the Aegean region. Int. J. Earth Sci. 2010, 99, 109-138.

27. Jolivet, L.; Faccenna, C.; Huet, B.; Labrousse, L.; Le Pourhiet, L.; Lacombe, O.; Lecomte, E.; Burov, E.; Denèle, Y.; Brun, J.P.; et al. Aegean tectonics: Strain localization, slab tearing and trench retreat. Tectonophysics 2012, doi:10.1016/j.tecto.2012.06.011.

28. Ring, U.; Glodny, J.; Will, T.; Thomson, S. The Hellenic subduction system: high-pressure metamorphism, exhumation, normal faulting, and large-scale extension. Ann. Rev. Earth Planet. Sci. 2010, 38, 45-76.

29. Van Hinsbergen, D.J.J.; Hafkenscheid, E.; Spakman, W.; Meulenkamp, J.E.; Wortel, R. Nappe stacking resulting from subduction of oceanic and continental lithosphere below Greece. Geology 2005, 33, 325-328.

30. Brun, J.P.; Faccenna, C. Exhumation of high-pressure rocks driven by slab roll-back. Earth Planet. Sci. Lett. 2008, 272, 1-7.

31. Marchev, P.; Kaiser-Rohrmeier, M.; Heinrich, Ch.; Ovtcharova, M.; von Quadt, A.; Raicheva, R. Hydrothermal ore deposits related to post-orogenic extentional magmatism and core complex formation: the Rhodope Massif of Bulgaria and Greece. Ore Geol. Rev. 2005, 27, 53-89.

32. Wüthrich, E.D. Low Temperature Thermochronology of the Northern Aegean Rhodope Massif. Ph.D. Thesis, ETH Zürich, Zürich, Switzerland, 2009.

33. Foster, M.; Lister, G. Core-complex-related extension of the Aegean lithosphere initiated at the Eocene-Oligocene transition. J. Geophys. Res. Solid Earth 2009, 114, B02401:1-B02401:36, doi:10.1029/2007JB005382.

34. Burg, J.P. Rhodope: From Mesozoic convergence to Cenozoic extension. Review of petro-structural data in the geochronological frame. J. Virtual Explor. 2012, 42, Paper 1.

35. Pe-Piper, G.; Piper, D.J.W. Unique features of the Cenozoic igneous rocks of Greece. In Postcollisional Tectonics and Magmatism in the Mediterranean Region and Asia; Dilek, Y., Pavlides, S., Eds.; Geological Society of America Special Paper 409; The Geological Society of America: Boulder, CO, USA, 2006; pp. 259-282.

36. Innocenti, F.; Kolios, N.; Manetti, O.; Mazzuoli, R.; Peccerillo, G.; Rita, F.; Villari, L. Evolution and geodynamic significance of the Tertiary orogenic volcanism in northeastern Greece. Bull. Vulcanol. 1984, 47, 25-37.

37. Perugini, D.; Poli, G.; Christofides, G.; Eeftheriadis, G.; Koroneos, A.; Soldatos, T. Mantle-derived and crustal melts dichotomy in northern Greece: spatiotemporal and geodynamic implications. Geol. J. 2004, 39, 63-80.

38. Fytikas, M.; Innocenti, F.; Manetti, O.; Mazzuoli, R.; Peccerillo, G.; Villari, L. Tertiary to Quaternary evolution of volcanism in the Aegean region. In The Geological Evolution of the Eastern Mediterranean; Geological Society Special Publications No. 17; Dixon, J.E., Robertson, A.H.F., Eds.; The Geological Society: London, UK, 1984; pp. 687-699. 
39. Melfos, V.; Vavelidis, M.; Christofides, G.; Seidel, E. Origin and evolution of the Tertiary Maronia porphyry copper-molybdenum deposit, Thrace, Greece. Miner. Depos. 2002, 37, 648-668.

40. Moritz, R.; Márton, I.; Ortelli, M.; Marchev, P.; Voudouris, P.; Bonev, N.; Spikings, R.; Cosca, M. A Review of Age Constraints of Epithermal Precious and Base Metal Deposits of the Tertiary Eastern Rhodopes: Coincidence with Late Eocene-Early Oligocene Tectonic Plate Reorganization along the Tethys. In Scientific Annals of the School of Geology AUTH, Proceedings of the 19th Congress of the Carpathian-Balkan Geological Association, Thessaloniki, Greece, 23-26 September 2010; pp. 351-358.

41. Arvanitidis, N.D.; Constantinides, D. Base and precious metal sulfide mineralization of the Greek Rhodope Massif. Geol. Rhodop. 1989, 1, 298-305.

42. Arikas, K.; Voudouris, P. Hydrothermal alterations and mineralizations of magmatic rocks in the southern Rhodope Massif. Acta Volcan. 1998, 10, 353-365.

43. Voudouris, P. Comparative mineralogical study of Tertiary Te-rich epithermal and porphyry systems in northeastern Greece. Mineral. Petrol. 2006, 87, 241-275.

44. Márton, I.; Moritz, R.; Spikings, R. Application of low-temperature thermochronology to hydrothermal ore deposits: Formation, preservation and exhumation of epithermal gold systems from the Eastern Rhodopes, Bulgaria. Tectonophysics 2010, 483, 240-254.

45. Eliopoulos, D.; Kilias, S.P. Marble-hosted submicroscopic gold mineralization at Asimotrypes area, Mount Pangeon, southern Rhodope Core Complex, Greece, Econ. Geol. 2011, 106, 751-780.

46. Voudouris, P.; Tarkian, M.; Arikas, K. Mineralogy of telluride-bearing epithermal ores in Kassiteres-Sappes area, western Thrace, Greece. Mineral. Petrol. 2006, 87, 31-52.

47. Ortelli, M. Tertiary Porphyry and Epithermal Association of the Sapes/Kassiteres District, Eastern Rhodopes, Greece. Master's Thesis, University of Geneva, Geneva, Switzerland, October 2009.

48. Ortelli, M.; Moritz, R.; Voudouris, P.; Spangenberg, J. Tertiary porphyry and epithermal association of the Sapes-Kassiteres district, eastern Rhodopes, Greece. In Smart Science for Exploration and Mining, Proceedings of the 10th Biennial SGA Meeting, Townsville, Australia, 17-20 August 2009; Williams, P.J., Ed.; Economic Geology Research Unit, James Cook University: Douglas, Australia, 2009; pp. 536-538.

49. Papadopoulou, L.; Christofides, G.; Koroneos, A.; Bröcker, M.; Soldatos, T.; Eleftheriadis, G. Evolution and origin of the Maronia pluton, Thrace, Greece. Bull. Geol. Soc. Greece 2004, 36, $568-577$.

50. Le Bas, M.J.; Le Maitre, R.W.; Streckeisen, A.; Zenettin, B. A chemical classification of volcanic rocks based on the total alkali-silica diagram. J. Petrol. 1986, 27, 745-750.

51. Irvine, T.N.; Baragar, W.R.A. A guide to the chemical classification of the common rocks. Can. J. Earth Sci. 1971, 8, 523-548.

52. Streckeisen, A.; Le Maitre, R.W. A chemical approximation to the modal QAPF classification of the igneous rocks. N. Jb. Miner. Abh. 1979, 136, 169-206.

53. Eleftheriadis, G. Petrogenesis of the Oligocene volcanics from the Central Rhodope massif (N. Greece). Eur. J. Mineral. 1995, 7, 1169-1182.

54. Voudouris, P.; Melfos, V. Aluminum-phosphate-sulfate (APS) minerals in the sericitic-advanced argillic alteration zone of the Melitena porphyry-epithermal $\mathrm{Mo}-\mathrm{Cu}- \pm \mathrm{Au} \pm \mathrm{Re}$ prospect, western Thrace, Greece. N. Jb. Mineral. Abh. 2012, 190, 11-27. 
55. Gustafson, L.B.; Hunt, J.P. The porphyry copper deposit at El Salvador, Chile. Econ. Geol. 1975, 70, 857-912.

56. Dilles, J.H.; Einaudi, M.T. Wall-rock alteration and hydrothermal flow paths about the Ann-Mason porphyry copper deposit, Nevada-A 6-km vertical reconstruction. Econ. Geol. 1992, 87, 1963-2001.

57. Muntean, J.L.; Einaudi, M.T. Porphyry-epithermal transition: Maricunga belt, Northern Chile. Econ. Geol. 2001, 96, 743-772.

58. Seedorff, E., Dilles, J.H.; Proffett, J.M.; Einaudi, M.T.; Zurcher, L.; Stavast, W.J.A.; Johnson, D.A.; Barton, M.D. Porphyry deposits: Characteristics and origin of hypogene features. Econ. Geol. 2005, 100, 251-298.

59. Arancibia, O.N.; Clark, A.H. Early magnetite-amphibole-plagioclase alteration-mineralization in the Island Copper porphyry copper-gold-molybdenum deposit, British Columbia. Econ. Geol. 1996, 91, 402-438.

60. Katirtzoglou, C. The Metallogenesis of the Tertiary Sulfide Mineralization of the Essymi Region, Evros County. Ph.D. Thesis, University of Athens, Athens, Greece, 1986.

61. Voudouris, P.; Alfieris, D. New porphyry- $\mathrm{Cu} \pm$ Mo occurrences in northeastern Aegean/Greece: Ore mineralogy and transition to epithermal environment. In Mineral Deposit Research: Meeting the Global Challenge, Proceedings of the 8th Biennial SGA Meeting, Beijing, China, 18-21 August 2005; Mao, J., Bierlein, F.P., Eds.; Springer: Berlin, Germany, 2005, pp. 473-476.

62. Fornadel, A.P.; Voudouris, P.; Spry, P.G.; Melfos, V. Mineralogical, stable isotope and fluid inclusion studies of spatially related porphyry $\mathrm{Cu}-\mathrm{Mo}$ and epithermal $\mathrm{Au}-\mathrm{Te}$ mineralization, Fakos Peninsula, Limnos Island, Greece. Mineral. Petrol. 2012, 105, 85-111.

63. Todorov, T. Genetic types and metal-formation of molybdenum mineralization in Bulgaria. J. Southeast Asian Earth Sci. 1973, 8, 307-311.

64. Tarkian, M.; Stribny, B. Platinium group elements in porphyry copper deposits: A reconnaissance study. Mineral. Petrol. 1999, 65, 161-183.

65. Strashimirov, S.; Petrunov, R.; Kanazirski, M. Porphyry-copper mineralization in the central Srednogorie zone, Bulgaria. Miner. Depos. 2002, 37, 587-598.

66. Tockmakchieva, M. Valuable minor components in the composition of porphyry copper deposits. In Annual Report of the University of Mining and Geology "St. Ivan Rilski" Volume 45, Part I, Geology, Sofia; University of Mining and Geology "St. Ivan Rilski": Sofia, Bulgaria, 2002; pp. 71-75.

67. Todorov, T.; Staikov, M. Rhenium content in molybdenite from ore mineralizations in Bulgaria. Geol. Balc. 1985, 15, 45-58.

68. Armstrong, R.; Kozelj, D.; Herrington, R. The Majdanpek Cu-Au deposit of eastern Serbia, a review. In Super Porphyry Copper and Gold Deposits: A Global Perspective; Porter, T.M., Ed.; PGC Publishing: Adelaide, Australia, 2005; Volume 2, pp. 453-466.

69. Pavicevic, M.K.; Krajnovic, D.; Cvetcovic, L.; Grzetic, I. The Trace Elements in Chalcopyrite and Pyrite in the Bor Copper Deposits. In Proceedings of XV Congress of the Carpatho-Balcan Geological Association, Athens, Greece, 17-20 September 1995; pp. 804-807.

70. Wanhainen, C.; Billström, K.; Martinsson, O. Copper and gold distribution at the Aitik deposit, Gällivare area, northern Sweden. Appl. Earth Sci. 2003, 112, 260-267. 
71. Nigatu, W. Rhenium in the Aitik Cu-Au-Ag-(Mo) Deposit. Master's Thesis, Lulea University of Technology, Lulea, Sweden, December 2011.

72. Sotnikov, V.I.; Berzina, A.N.; Economou-Eliopoulos, M.; Eliopoulos, D.G. Palladium, platinum and gold distribution in porphyry $\mathrm{Cu} \pm$ Mo deposits of Russia and Mongolia. Ore Geol. Rev. 2001, $18,95-111$.

73. Singer, D.A.; Berger, V.I.; Moring, B.B. Porphyry Copper Deposits of the World: Database and Grade and Tonnage Models; Open-File Report 2008-1155; U.S. Geological Survey: Reston, VA, USA, 2008. Available online: http://pubs.usgs.gov/of/2008/1155/ (accessed on 6 June 2008).

74. Aminzadeh, B.; Shahabpour, J.; Maghami, M. Variation of rhenium contents in molybdenites from the Sar Cheshmeh porphyry Cu-Mo deposit in Iran. Res. Geol. 2011, 61, 290-295.

75. Gilles, D.L.; Schilling, J.H. Variation in Rhenium Content of Molybdenite. In Proceedings of the 24th International Geological Congress Section 10, Montreal, Canada, 1972; pp. 145-152.

76. Tarkian, M.; Koopmann, G. Platinum-group minerals in the Santo Thomas II (Philex), porphyry copper-gold deposit Luzon Island, Philippines. Miner. Depos. 1995, 30, 39-47.

77. Marinov, D. Re-Os molybdenite geochronology from Michiquillay and Galeno porphyry copper deposits, Cajamarca, Perú. In Let's Talk Ore Deposits, Proceedings of the 11th Biennial SGA Meeting, Antofagasta, Chile, 26-29 September 2011; Barra, F., Reich, M., Campos, E., Tornos, F., Eds.; Universidad Católica del Norte: Antofagasta, Chile, 2011.

78. Drábek, M.; Rieder, M.; Böhmová, V. The Re-Mo-S system: New data on phase relations between 400 and $1200{ }^{\circ}$ C. Eur. J. Mineral. 2010, 22, 479-484.

79. Kosler, J.A.; Simonetti, A.; Sylvester, P.J.; Cox, R.A.; Tubrett, M.N.; Wilton, D.H.C. Laser ablation ICP-MS measurements of Re/Os in molybdenite and implications for Re-Os geochronology. Can. Mineral. 2003, 41, 307-320.

80. Selby, D.; Creaser, R.A. Re-Os geochronology and systematics in molybdenite from the Endako porphyry molybdenum deposit, British Columbia, Canada. Econ. Geol. 2001, 96, 197-204.

81. Selby, D.; Creaser, R.A. Macroscale NTIMS and mircoscale LA-MC-ICP-MS Re-Os isotopic analysis of molybdenite: Testing spatial restriction for reliable Re-Os age determinations, and implications for the decoupling of Re and Os within molybdenite. Geochim. Cosmochim. Acta 2004, 68, 3897-3908.

82. Grabezhev, A.I.; Shagalov, E.S. Rhenium distribution in molybdenite: Results of microprobe scanning (copper porphyry deposits, the Urals). Dokl. Earth Sci. 2010, 431, 351-355.

83. Mao, J.; Zhang, Z.; Zhang, Z.; Du, A. Re-Os isotopic dating of molybdenites in the Xiaoliugou W(Mo) deposit in the northern Qilian Mountains and its geological significance. Geochim. Cosmochim. Acta 1999, 63, 1815-1818.

84. Blevin, P.L. The primacy of magma compositions in determining the Re and $\mathrm{W}$ contents of molybdenite. In Proceedings of the 24th International Applied Geochemistry Symposium, Fredericton, Canada, 1 June to 4 June 2009; pp. 119-122.

85. Selby, D.; Creaser, R.A.; Hart, C.J.R.; Rombach, C.S.; Thompson, J.F.H.; Smith, T.; Bakke, A.; Goldfarb, R. Absolute timing of sulfide and gold mineralization: A comparison of Re-Os molybdenite and Ar-Ar mica methods from the Tintina Gold Belt, Alaska. Geology 2002, 30, 791-794. 
86. Selby, D.; Creaser, R.A.; Heaman, L.M.; Hart, C.J.R. Re-Os and U-Pb geochronology of the Clear Creek, Dublin Gulch, and Mactung deposits, Tombstone Gold Belt, Yukon, Canada: absolute timing relationships between plutonism and mineralization. Can. J. Earth Sci. 2003, 40, 1839-1852.

87. Jones, C.E.; Tarney, J.; Baker, J.H.; Gerouki, F. Tertiary granitoids of Rhodope, northern Greece: Magmatism related to extensional collapse of the Hellenic Orogen? Tectonophysics 1992, 210 , 295-314.

88. Christofides, G.; Soldatos, T.; Elefthertiadis, G.; Koroneos, A. Chemical and isotopic evidence for source contamination and crustal assimilation in the Hellenic Rhodope plutonic rocks. Acta Volcanol. 1998, 10, 305-318.

89. Altherr, R.; Siebel, W. I-type plutonism in a continental back-arc setting: Miocene granitoids and monzonites from the central Aegean Sea, Greece. Contrib. Mineral. Petrol. 2002, 143, 397-415.

90. Skarpelis, N.; Tsikouras, B.; Pe-Piper, G. The Miocene igneous rocks in the Basal Unit of Lavrion (SE Attica, Greece): Petrology and geodynamic implications. Geol. Mag. 2008, 145, 1-15.

91. Christofides, G.; Soldatos, T.; Koroneos, A. Geochemistry and evolution of the Fanos granite, N. Greece. Mineral. Petrol. 1990, 43, 49-63.

92. Iglseder, C.; Grasemann, B.; Schneider, D.A.; Petrakakis, K.; Miller, C.; Klötzlid, U.S.; Thöni, M.; Zámolyi, A.; Rambousek, C. I and S-type plutonism on Seriphos (W-Cyclades, Greece). Tectonophysics 2009, 473, 69-83.

93. Xiong, Y.; Wood, S.A. Experimental determination of the solubility of $\mathrm{ReS}_{2}$ and the $\mathrm{Re}-\mathrm{ReO}_{2}$ buffer assemblage and transport of rhenium under supercritical conditions. Geochem. Trans. 2002, 3, 1-10.

94. Pe-Piper, G.; Piper, D.J. The Igneous Rocks of Greece: The Anatomy of an Orogen; Pe-Piper, G., Piper, D.J.W., Eds.; Beiträge zur regionalen Geologie der Erde 30; Gebruder Borntraeger: Berlin, Germany, 2002.

95. Del Moro, A.; Innocenti, F.; Kyriakopoulos, C.; Manetti, P.; Papadopoulos, P. Tertiary granitoids from Thrace (Northern Greece): Sr isotopic and petrochemical data. N. Jb. Miner. Abh. 1988, 159, 113-135.

96. Pe-Piper, G.; Piper, D.J.; Koukouvelas, I.; Dolansky, L.M.; Kokkalas, S. Postorogenic shoshonitic rocks and their origin by melting underplated basalts: The Miocene of Limnos, Greece. Geol. Soc. Am. Bull. 2009, 121, 39-54.

97. Kroll, T.; Muller, D.; Seifert, T.; Herzig, P.M.; Schneider, A. Petrology and geochemistry of the shoshonite-hosted Skouries porphyry $\mathrm{Cu}-\mathrm{Au}$ deposit, Chalkidiki, Greece. Miner. Depos. 2002, 37, 137-144.

98. Richards, J.P. Postsubduction porphyry $\mathrm{Cu}-\mathrm{Au}$ and epithermal $\mathrm{Au}$ deposits: Products of remelting of subduction-modified lithosphere. Geology 2009, 37, 247-250.

99. Richards, J.P. Magmatic to hydrothermal metal fluxes in convergent and collided margins. Ore Geol. Rev. 2011, 40, 1-26.

100. Li, N.; Chen, Y.J.; Santosh, M.; Yao, J.M.; Sun, Y.L.; Li, J. The 1.85 Ga Mo mineralization in the Xiong'er Terrane, China: Implications for metallogeny associated with assembly of the Columbia supercontinent. Precambrian Res. 2011, 186, 220-232. 
101. Jensen, E.P.; Barton, M.D. Gold deposits related to alkaline magmatism. Rev. Econ. Geol. 2000, 13, 279-314.

102. Sun, W.; Arculus, R.J.; Bennett, V.C.; Eggins, S.M.; Binns, R.A. Evidence for rhenium enrichment in the mantle wedge from submarine arc like volcanic glasses (Papua New Guinea). Geology 2003, 31, 845-848.

103. Tessalina, S.G.; Yudovskaya, M.A.; Chaplygin, I.V.; Birck, J.-L.; Capmas, F. Sources of unique rhenium enrichment in fumaroles and sulphides at Kudryavy volcano. Geochim. Cosmochim. Acta 2008, 72, 889-909.

104. Ciobanu, C.L.; Cook, N.J.; Damian, G.; Damian, F. Telluride and sulfosalt associations at Săcărîmb. In Au-Ag-Telluride Deposits of the Golden Quadrilateral, Apuseni Mts., Romania; Cook, N.J., Ciobanu, C.L., Eds.; International Field Workshop of IGCP Project 486; IAGOD Guidebook Series 12; Romanian Geological Survey: Alba Iulia, Romania, 2004; pp. 145-186.

105. Cook, N.J.; Ciobanu, C.L.; Damian, G.; Damian, F. Tellurides and sulfosalts from deposits in the Golden Quadrilateral. In Au-Ag-Telluride Deposits of the Golden Quadrilateral, Apuseni Mts., Romania; Cook, N.J., Ciobanu, C.L., Eds.; International Field Workshop of IGCP Project 486; IAGOD Guidebook Series 12; Romanian Geological Survey: Alba Iulia, Romania, 2004; pp. 111-144.

106. Cook, N.J.; Ciobanu, C.L. Bismuth tellurides and sulfosalts from the Larga hydrothermal system, Metaliferi Mts, Romania: Paragenesis and genetic significance. Mineral. Mag. 2004, 68, 301-321.

107. Harris, C.R.; Pettke, T.; Heinrich, C.A.; Rosu, E.; Woodland, S.; Fry, B. Tethyan mantle metasomatism creates subduction geochemical signatures in non-arc $\mathrm{Cu}-\mathrm{Au}-\mathrm{Te}$ mineralizing magmas, Apuseni Mountains (Romania). Earth Planet. Sci. Lett. 2013, 366, 122-136.

108. Tarkian, M.; Eliopoulos, D.G.; Economou-Eliopoulos, M. Mineralogy of precious metals in the Skouries porphyry copper deposit, Northern Greece. N. Jb. Miner. Abh. 1991, 12, 529-537.

109. Spry, P.G.; Foster, F.; Truckle, J.S.; Chadwick, T.H. The mineralogy of the Golden Sunlight gold-silver telluride deposit, Whitehall, Montana, U.S.A. Mineral. Petrol. 1997, 59, 143-164.

110. LeFort, D.; Hanley, J.; Guillong, M. Subepithermal Au-Pd mineralization associated with an alkalic porphyry $\mathrm{Cu}-\mathrm{Au}$ deposit, Mount Milligan, Quesnel Terrane, British Columbia, Canada. Econ. Geol. 2011, 106, 781-808.

111. Zimmerman, A.; Stein, H.J.; Hannah, J.L.; Kozelj, D.; Bogdanov, K.; Berza, T. Tectonic configuration of the Apuseni-Banat-Timrok-Srednogorie belt, Balkans-South Carpathians, constrained by high precision Re-Os. Miner. Depos. 2008, 43, 1-21.

112. Leng, C.-B.; Zhang, X.-C.; Hu, R.-Z.; Wang, S.-X.; Zhong, H.; Wang, W.-Q.; Bi, X.-W. Zircon $\mathrm{U}-\mathrm{Pb}$ and molybdenite Re-Os Geochronology and $\mathrm{Sr}-\mathrm{Nd}-\mathrm{Pb}-\mathrm{Hf}$ isotopic constraints on the genesis of the Xuejiping porphyry copper deposit in Zhongdian, Northwest Yunnan, China. J. Asian Earth Sci. 2012, 60, 31-48.

(C) 2013 by the authors; licensee MDPI, Basel, Switzerland. This article is an open access article distributed under the terms and conditions of the Creative Commons Attribution license (http://creativecommons.org/licenses/by/3.0/). 